\title{
Chiral flavor violation from extended gauge mediation
}

\author{
Jared A. Evans, ${ }^{a}$ David Shih $^{b}$ and Arun Thalapillil ${ }^{b}$ \\ ${ }^{a}$ Department of Physics, University of Illinois at Urbana-Champaign, \\ Urbana, IL 61801, U.S.A. \\ ${ }^{b}$ NHETC, Department of Physics and Astronomy, \\ Rutgers University, Piscataway, NJ 08854, U.S.A. \\ E-mail: evansja@illinois.edu, dshih@physics.rutgers.edu, \\ thalapillil@physics.rutgers.edu
}

AbStRact: Models of extended gauge mediation, in which large $A$-terms arise through direct messenger-MSSM superpotential couplings, are well-motivated by the discovery of the $125 \mathrm{GeV}$ Higgs. However, since these models are not necessarily MFV, the flavor constraints could be stringent. In this paper, we perform the first detailed and quantitative study of the flavor violation in these models. To facilitate our study, we introduce a new tool called FormFlavor for computing precision flavor observables in the general MSSM. We validate FormFlavor and our qualitative understanding of the flavor violation in these models by comparing against analytical expressions. Despite being non-MFV, we show that these models are protected against the strongest constraints by a special flavor texture, which we dub chiral flavor violation $(\chi \mathrm{FV})$. This results in only mild bounds from current experiments, and exciting prospects for experiments in the near future.

Keywords: Supersymmetric Standard Model, Rare Decays, B-Physics, Kaon Physics

ARXIV EPRINT: 1504.00930 


\section{Contents}

1 Introduction 1

2 Chiral flavor violation and EGMSB 5

2.1 Chiral flavor violation 5

$\begin{array}{lll}2.2 & \chi \mathrm{FV} \text { in type I squark models } & 7\end{array}$

3 Flavor observables and constraints on EGMSB $\quad 9$

3.1 Q-class models $\quad 11$

$\begin{array}{lll}3.1 .1 & \text { Meson mixing } & 11\end{array}$

$\begin{array}{lll}3.1 .2 \quad K^{ \pm} \rightarrow \pi^{ \pm} \nu \bar{\nu} & 14\end{array}$

$\begin{array}{lll}3.1 .3 \quad b \rightarrow s \gamma \text { and } b \rightarrow d \gamma & 15\end{array}$

$\begin{array}{lll}3.1 .4 & B_{q} \rightarrow \mu^{+} \mu^{-} & 17\end{array}$

3.2 U-class models 20

4 Conclusions 20

4.1 Summary and discussion 20

$\begin{array}{lll}4.2 & \text { Future constraints } & 21\end{array}$

4.3 Future directions 23

A Details of the deformation and parameter scan 24

B Details of FormFlavor $\quad 26$

$\begin{array}{ll}\text { C Soft terms for type I squark models } & 27\end{array}$

D Formulas for flavor observables $\quad \mathbf{2 9}$

D.1 Meson Mixing $\Delta m_{X} \quad 30$

D.2 $K^{ \pm} \rightarrow \pi^{ \pm} \nu \bar{\nu}$

D.3 $b \rightarrow s \gamma$ and $b \rightarrow d \gamma \quad 32$

D.4 $B_{s} \rightarrow \mu^{+} \mu^{-}$and $B_{d} \rightarrow \mu^{+} \mu^{-}$

E Rank $1 \chi$ FV loop functions $\quad 33$

\section{Introduction}

The SUSY flavor problem is a serious challenge for models of weak-scale supersymmetry. (For a review and original references, see [1,2].) Soft SUSY-breaking introduces many sources of flavor violation beyond the Standard Model Yukawas. Generic points of the MSSM parameter space are ruled out by myriad precision flavor constraints, such as neutral 
meson mixing and $b \rightarrow s \gamma$. Clearly, the underlying mechanism that generates these soft SUSY-breaking parameters from the hidden sector must be quite special. Historically, the lore has been that the mediation of SUSY-breaking must either be flavor-blind or obey the minimal flavor-violating (MFV) ansatz. Gauge mediated SUSY breaking (GMSB), which is manifestly flavor-blind, is one of the simplest solutions to the SUSY flavor problem (see [3] for a review and original references).

The 2012 discovery of a Standard Model-like Higgs with a mass near $125 \mathrm{GeV}[4,5]$ presents interesting challenges for models of GMSB, especially in the MSSM, where a $125 \mathrm{GeV}$ Higgs implies either very heavy stops $\gtrsim 10 \mathrm{TeV}$ or large (multi-TeV) stop $A$ terms [6-10]. The heavy stop scenario is more fine-tuned and less interesting from both an experimental and theoretical point of view. The large $A$-term scenario allows for light stops, but a mechanism is required to generate these $A$-terms, which are absent at the messenger scale in GMSB.

Large $A$-terms can arise if the usual GMSB framework is extended to also include direct MSSM-messenger superpotential couplings. Using light $(\sim 1 \mathrm{TeV})$ stops and large $A$-terms, these models of extended GMSB (EGMSB) can give rise to the observed Higgs mass at fine-tuning levels close to the best achievable within the MSSM [11-28]. However, since these MSSM-messenger superpotentials are typically flavorful, they are in danger of reintroducing the SUSY flavor problem. Previous works on EGMSB have either assumed perfect alignment with the third-generation (to get a large stop $A$-term), or considered additional model building (such as Froggatt-Nielsen mechanisms) to ensure this alignment $[18,20,21,29-34]$. In this paper, we will not presume any such alignment, and we will instead perform the first comprehensive study of the general flavor constraints on EGMSB models for the Higgs mass.

The precursor to this work was the complete classification of all renormalizable EGMSB couplings consistent with perturbative SU(5) unification provided in [20] (see also [18]). By turning on one coupling at a time (perfect alignment with the third generation was assumed) and imposing the Higgs mass constraint, the landscape of EGMSB models was surveyed for their phenomenology and fine-tuning. It was shown that the EGMSB models that exhibit the smallest tuning are of the form,

$$
W \ni \kappa_{3} Q_{3} \Phi \tilde{\Phi}, \quad W \ni \kappa_{3} \bar{U}_{3} \Phi \tilde{\Phi}, \quad W \ni \kappa_{3} Q_{3} H_{u} \Phi, \quad \text { or } \quad W \ni \kappa_{3} \bar{U}_{3} H_{u} \Phi .
$$

Here $\Phi, \tilde{\Phi}$ are messenger fields transforming in appropriate representations of the SM gauge group. The first two (second two) models involve a single MSSM field (two MSSM fields) and so they were classified as "type I" ("type II") models.

Notice that the least fine-tuned models in (1.1) are all flavorful. Type I Higgs models, i.e., $W \ni \kappa H_{u} \Phi \tilde{\Phi}$, while MFV, are more finely-tuned due to the "little $A / m_{H}^{2}$ problem" [13]. These models have an irreducible contribution to $m_{H_{u}}^{2}=A_{\tilde{t}}^{2}+\ldots$ where $A_{\tilde{t}}$ is the stop $A$-term; thus a large $A_{\tilde{t}}$ is tied to a large $m_{H_{u}}^{2}$ and one does not improve tuning in these models by increasing the $A$-terms.

In this paper, we will study the effects that arise when the couplings of (1.1) are no longer required to align perfectly with the third-generation. For simplicity, we will 
specialize to a pair of representative type I $Q$-class and $U$-class models, the models I.9 and I.13 from [20],

$$
W \ni \kappa_{i} Q_{i} \Phi_{D} \Phi_{L}, \quad W \ni \kappa_{i} \bar{U}_{i} \Phi_{D_{1}} \Phi_{D_{2}}
$$

where the messengers $\Phi_{D}$ and $\Phi_{L}$ have the SM gauge quantum numbers of the $D$ and $L$ matter fields. We have verified explicitly that the other type I squark models are very similar, both qualitatively and quantitatively. For reasons we will explain shortly, we expect the type II models are also qualitatively similar in their features. We will not consider the effect of turning on multiple EGMSB couplings, or couplings involving lepton flavor violation. Such couplings can be forbidden by appropriate choices of discrete symmetries. Finally, to focus exclusively on the SUSY flavor problem, we will not consider CP violation in this work, i.e., all the couplings $\kappa_{i}$ are taken to be real in the mass basis of the standard model particles. The (possibly stringent) constraints from CP violating observables, such as $\epsilon_{K}$ and the neutron EDM, will be studied in a forthcoming publication [35].

The interactions in (1.2) result in flavor-violating contributions to the squark mass matrices

$$
\delta \mathcal{M}_{\tilde{u}}^{2}=\left(\begin{array}{c|c}
\delta m_{Q}^{2} & s_{\beta} v A_{\tilde{u}}^{\dagger} \\
\hline s_{\beta} v A_{\tilde{u}} & \delta m_{U}^{2}
\end{array}\right), \quad \delta \mathcal{M}_{\tilde{d}}^{2}=\left(\begin{array}{c|c}
\delta m_{Q}^{2} & c_{\beta} v A_{\tilde{d}}^{\dagger} \\
\hline c_{\beta} v A_{\tilde{d}} & \delta m_{D}^{2}
\end{array}\right)
$$

Here each block (clockwise from upper left: $L L, L R, R R, R L$ ) is a $3 \times 3$ matrix. These should be added to the flavor-conserving GMSB contributions and the supersymmetric contributions, and together they contribute to precision flavor observables through a variety of one-loop diagrams involving the squarks and other superpartners. The calculation of these one-loop diagrams for general MSSM spectra is a tedious and laborious task, ideally suited for a computer program. There are several such programs that are publicly available; unfortunately, we found that they were all unsuitable for our purpose. These programs either assumed MFV, did not have a sufficiently broad list of flavor observables, were numerically unstable, or were found to have bugs, likely introduced in transcribing formulas by hand from the literature.

As a result, we found it necessary to develop a new tool called FormFlavor for the study of flavor physics. FormFlavor takes general MSSM spectra and computes the contributions to the various flavor observables shown in table 1. The novel aspect of FormFlavor is that the computation of one-loop Wilson coefficients in the MSSM is done completely from scratch, using the general-purpose packages FeynArts [36] and FormCalc [37]. ${ }^{1}$ This avoids the problems associated with transcribing formulas from the literature, and it facilitates the inclusion of additional flavor observables in an automated and modular way. We intend to make FormFlavor publicly available; its usage and validation will be described in an upcoming publication [39].

Starting from the points of reduced tuning identified in [20], we will turn on $\kappa_{1,2}$ and use FormFlavor to investigate the constraints from precision flavor observables. Given that

\footnotetext{
${ }^{1}$ While this work was in preparation, a new tool Flavorkit [38] was published with a very similar approach to calculating flavor observables from scratch. FlavorKit aims to be even more general, in that it can derive the one-loop Wilson coefficients for a general model, not just the MSSM. It would be interesting to compare the two codes in detail.
} 


\begin{tabular}{|c|c|c|}
\hline Observable & Experiment & SM prediction \\
\hline$\Delta m_{K}$ & $(3.484 \pm 0.006) \times 10^{-15} \mathrm{GeV}$ & - \\
\hline$\Delta m_{B_{d}}$ & $(3.36 \pm 0.02) \times 10^{-13} \mathrm{GeV}$ & $(3.56 \pm 0.60) \times 10^{-13} \mathrm{GeV}[40]$ \\
\hline$\Delta m_{B_{s}}$ & $(1.169 \pm 0.0014) \times 10^{-11} \mathrm{GeV}$ & $(1.13 \pm 0.17) \times 10^{-11} \mathrm{GeV}[40]$ \\
\hline$\Delta m_{D}$ & $\left(6.2_{-2.8}^{+2.7}\right) \times 10^{-15} \mathrm{GeV}$ & - \\
\hline$B r\left(K^{+} \rightarrow \pi^{+} \nu \bar{\nu}\right)$ & $(1.7 \pm 1.1) \times 10^{-10}$ & $(7.8 \pm 0.8) \times 10^{-11}[41]$ \\
\hline$B r\left(B \rightarrow X_{s} \gamma\right)$ & $(3.40 \pm 0.21) \times 10^{-4}$ & $(3.15 \pm 0.23) \times 10^{-4}[42]$ \\
\hline$B r\left(B \rightarrow X_{d} \gamma\right)$ & $(1.41 \pm 0.57) \times 10^{-5}[43,44]$ & $\left(1.54_{-0.31}^{+0.26}\right) \times 10^{-5}[44]$ \\
\hline$B r\left(B_{s} \rightarrow \mu^{+} \mu^{-}\right)$ & $(2.9 \pm 0.7) \times 10^{-9}[45]$ & $(3.65 \pm 0.23) \times 10^{-9}[46]$ \\
\hline$B r\left(B_{d} \rightarrow \mu^{+} \mu^{-}\right)$ & $\left(3.6_{-1.4}^{+1.6}\right) \times 10^{-10}[45]$ & $(1.06 \pm 0.09) \times 10^{-10}[46]$ \\
\hline
\end{tabular}

Table 1. Current experimental values based on PDG and HFAG fits $[47,48]$ except where noted. No reliable theoretical prediction for $\Delta m_{D}$ currently exists. Although literature on the subject exists, we do not use a theoretical prediction for $\Delta m_{K}$ (see the discussion of $\Delta m_{K}$ in section 4.2 for more details).

they are not MFV, one might expect these constraints to be extremely stringent. Generic operator bounds put the scale of flavor violation at $20 \mathrm{PeV}$ or higher from $K-\bar{K}$ and $D-\bar{D}$ mixing [2]. Even in SUSY, where one benefits from loop factors, etc., the bounds are close to $500 \mathrm{TeV}$ (without using $\mathrm{CP}$ violation) [49, 50]. However, we will find that in these EGMSB models, the limits from flavor-violation are extremely mild - to the point that $\kappa_{1,2,3}$ can all be the same size and yet the model is not ruled out by flavor!

Much of this paper will be devoted to identifying the reasons for these surprisingly mild flavor constraints. Although the overall heavier mass scale required to raise the Higgs mass plays a role, the most important reason is the fact that the EGMSB models (1.1) only violate flavor through a spurion of either $\mathrm{SU}(3)_{Q}$ or $\mathrm{SU}(3)_{U}$, but not both. Because of this chiral flavor violation $(\chi \mathrm{FV})$, EGMSB models have a novel flavor texture - flavor violation primarily occurs only in either the left-chiral sector of the squark mass-squared matrix (for the $Q$-class models) or the right-chiral sector of the squark mass-squared matrix (for the $U$-class models). Only via communication through the SM Yukawas can the other chiral sector feel the flavor violation. As it turns out, the most stringent flavor constraints, which come from $\Delta m_{K}$ and $\Delta m_{D}$, are vastly reduced when the flavor violation is restricted to only the left- or right-chiral sector. (It is also on these general grounds that we expect the type II models are similarly unconstrained by flavor, although it would be interesting to verify this in detail.) In a forthcoming work [51], we will study this new ansatz of $\chi \mathrm{FV}$ in more generality, along the lines of what has been done for MFV. $\chi \mathrm{FV}$ represents an interesting intermediate case between full flavor anarchy (which is known to be heavily constrained) and MFV (which is known to be basically unconstrained).

In order to validate and interpret our numerical findings, we will compare them against analytic expressions for the flavor observables. Historically, the mass insertion approximation (MIA) has been utilized to interpret the influence of flavor-violating squark masses on 
precision flavor observables (see e.g., [2] for a review of the MIA and original references). However, the utility of the MIA is limited when one or more of these mass insertions are $\mathcal{O}(1)$. Since that is precisely the interesting region of parameter space for our EGMSB models, the traditional MIA cannot be used here. Fortunately, in these EGMSB models there is another handle we can use to obtain an analytic understanding. From two powers of our anti-fundamental spurion, $\kappa_{a}$, we can construct an adjoint+singlet of $\mathrm{SU}(3)_{Q}$ or $\mathrm{SU}(3)_{U}$,

$$
K_{a b} \equiv \kappa_{a}^{*} \kappa_{b}
$$

This matrix $K$ governs all flavor violation from EGMSB. By exploiting the fact that it is only rank 1 , together with the special properties of the soft masses for the type I models, we are able to obtain analytic formulas for the flavor observables that treat the flavor violation exactly. Then the only expansion that we do is in $v / m_{\mathrm{SUSY}}$, which is still an excellent approximation in these models, even when the flavor violation is $\mathcal{O}(1)$. With this technique, we are able to make precise estimates of the supersymmetric contributions to flavor observables, validate our numerical results in detail, and understand their qualitative features.

Our paper is outlined as follows. In section 2, we describe the idea behind chiral flavor violation and how it applies to our models (1.1) in restricting the texture of the squark mass matrices. We then further restrict our attention to the type I squark models, and highlight some of their special features that will be useful in analyzing the flavor observables. In section 3 , we turn to a detailed study of the flavor constraints on the EGMSB models (1.2). We illustrate these constraints in the $\kappa_{1}-\kappa_{2}$ plane using a series of plots of flavor observables computed with FormFlavor. We also provide an analytic understanding of the features of these plots using the special properties of rank $1 \chi \mathrm{FV}$ and the type I squark models. Finally in section 4, we conclude with a brief summary of our results, and a discussion of the promising future prospects for precision flavor tests of these EGMSB models. In the appendices, we detail our parameter deformation and various subtleties that arise there, provide a brief description of FormFlavor (postponing a more detailed manual and validation for an upcoming work [39]), compile the necessary expressions for flavor observables using a uniform notation, and present some other formulas used in this work.

\section{Chiral flavor violation and EGMSB}

\subsection{Chiral flavor violation}

In the absence of Yukawa couplings, the Standard Model flavor symmetry group is

$$
G_{f}=\mathrm{SU}(3)_{Q} \times \mathrm{SU}(3)_{U} \times \mathrm{SU}(3)_{D} \times \mathrm{SU}(3)_{L} \times \mathrm{SU}(3)_{E}
$$

The assumption of MFV is that this flavor symmetry is broken only by the Yukawa couplings $y_{u}, y_{d}$ and $y_{\ell}$, which transform as $(\mathbf{3}, \overline{\mathbf{3}})$ under $\mathrm{SU}(3)_{U} \times \mathrm{SU}(3)_{Q}, \mathrm{SU}(3)_{D} \times \mathrm{SU}(3)_{Q}$, and $\mathrm{SU}(3)_{E} \times \mathrm{SU}(3)_{L}$, respectively. As described in the introduction, the chiral flavor violation $(\chi \mathrm{FV})$ ansatz goes beyond MFV, and postulates that, in addition to the Yukawas, the 
flavor symmetry is broken by additional spurions transforming only under a single $\mathrm{SU}(3)$ of the full flavor group.

The $\chi \mathrm{FV}$ ansatz results in a special texture of flavor violation in the MSSM, one which greatly suppresses the constraints from many flavor violating observables. In general, the $3 \times 3$ soft mass-squareds $m_{X}^{2}$ transform as an adjoint+singlet of $\mathrm{SU}(3)_{X}$, while the $A$-terms transform in the same way as the Yukawas. Using spurions from only a single $\mathrm{SU}(3)_{X}$, one can obtain flavor violation only in $m_{X}^{2}$. To obtain flavor violation in any of the other soft terms, one must involve the Yukawa couplings. Thus these other soft masses inherit an MFV-like suppression.

The cases of interest for this paper are when $X=Q$ or $U$. (We reserve a more general treatment for an upcoming publication [51].) Let us now examine these in more detail. To leading order in the Yukawa couplings, the symmetries of $Q$-class $\chi \mathrm{FV}$ imply that

$$
\delta m_{U}^{2}=y_{u} \Sigma y_{u}^{\dagger}, \quad \delta m_{D}^{2}=y_{d} \Sigma y_{d}^{\dagger}, \quad A_{\tilde{u}}=y_{u} \Gamma, \quad A_{\tilde{d}}=y_{d} \Gamma
$$

where $\Sigma$ and $\Gamma$ are built out of the $\mathrm{SU}(3)_{Q}$ spurions. Substituting these into (1.3), we have under the third-generation dominant approximation (whereby $y_{u}$ and $y_{d}$ are nonzero only in the 33 component):

$$
\begin{gathered}
\delta \mathcal{M}_{\tilde{u}}^{2} \approx\left(\begin{array}{ccc|ccc} 
& & & 0 & 0 & m_{t} \Gamma_{31}^{*} \\
& \delta m_{Q}^{2} & & 0 & 0 & m_{t} \Gamma_{32}^{*} \\
& & & 0 & 0 & m_{t} \Gamma_{33}^{*} \\
\hline 0 & 0 & 0 & 0 & 0 & 0 \\
0 & 0 & 0 & 0 & 0 & 0 \\
m_{t} \Gamma_{31} & m_{t} \Gamma_{32} & m_{t} \Gamma_{33} & 0 & 0 & y_{t}^{2} \Sigma_{33}
\end{array}\right) \\
\delta \mathcal{M}_{\tilde{d}}^{2} \approx\left(\begin{array}{ccc|ccc} 
& & & 0 & m_{b} \Gamma_{31}^{*} \\
& \delta m_{Q}^{2} & & 0 & 0 & m_{b} \Gamma_{32}^{*} \\
0 & 0 & 0 & 0 & 0 & m_{b} \Gamma_{33}^{*} \\
\hline 0 & 0 & 0 & 0 & 0 & 0 \\
m_{b} \Gamma_{31} & m_{b} \Gamma_{32} & m_{b} \Gamma_{33} & 0 & 0 & y_{b}^{2} \Sigma_{33}
\end{array}\right)
\end{gathered}
$$

We see that within the third-generation dominant approximation, no flavor violation appears in the RR block for either the up or down squark mass matrices. Also, the only flavor violation in the LR block involves the 3rd generation, and is $v / m_{\text {SUSY }}$ suppressed. These features greatly reduce the sensitivity of $Q$-class $\chi \mathrm{FV}$ to precision flavor constraints. As we will explain in more detail in section 3.1.1, what typically provide the most stringent flavor bounds on new physics (i.e., $\Delta m_{K}$ and $\Delta m_{D}$ ) involve the 1st and 2nd generations and involve simultaneous violation of flavor in both the left and right chiral sectors.

Meanwhile the $U$-class $\chi \mathrm{FV}$ models are even more insulated from constraints. Here, on symmetry grounds, and again to leading order in the Yukawas, we must have

$$
\delta m_{Q}^{2}=y_{u}^{\dagger} \Sigma y_{u}, \quad A_{\tilde{u}}=\Gamma y_{u}, \quad \delta m_{D}^{2}=A_{\tilde{d}}=0
$$


where now $\Sigma$ and $\Gamma$ are built out of the $\mathrm{SU}(3)_{U}$ spurions. Again substituting these into (1.3) in the third-generation dominant approximation, we find:

$$
\begin{aligned}
& \delta \mathcal{M}_{\tilde{u}}^{2} \approx\left(\begin{array}{ccc|ccc}
0 & 0 & 0 & 0 & 0 & 0 \\
0 & 0 & 0 & 0 & 0 & 0 \\
0 & 0 & y_{t}^{2} \Sigma_{33} & m_{t} \Gamma_{13}^{*} & m_{t} \Gamma_{23}^{*} & m_{t} \Gamma_{33}^{*} \\
\hline 0 & 0 & m_{t} \Gamma_{13} & & & \\
0 & 0 & m_{t} \Gamma_{23} & & \delta m_{U}^{2} & \\
0 & 0 & m_{t} \Gamma_{33} & & &
\end{array}\right),
\end{aligned}
$$

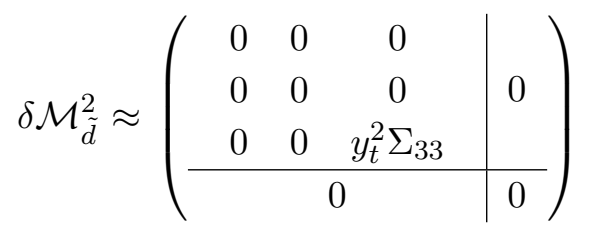

Thus there is no down-type flavor violation at all in the third-generation dominant approximation! As the majority of sensitive flavor probes involve the down sector, this eliminates almost all contributions to flavor observables. The only exception is $\Delta m_{D}$, which again has reduced sensitivity because there is no simultaneous left-right flavor violation in the 1st/2nd generations. (Down-type flavor observables can involve the up-squark RR block through chargino loops, but these must be mostly Higgsino-like, so they will be suppressed by Yukawa couplings.)

Finally, we should comment on the role of the RG. The RGEs from the messenger scale to the weak scale add more terms, but the symmetry-based arguments given above - which were truncated at leading order in the Yukawas - clearly continue to hold with the inclusion of higher orders in the Yukawas. In particular, terms that are zero in the third-generation dominant limit remain zero under RG evolution. For this reason, we do not need to concern ourselves with the details of RG running to gain a qualitative understanding of the EGMSB flavor-violating contributions to our flavor observables.

\section{$2.2 \chi$ FV in type I squark models}

The EGMSB models (1.1) are clearly examples of $Q$-class or $U$-class $\chi \mathrm{FV}$. In this subsection, we will discuss some further features that are specific to the type I squark models that will help us analyze the Wilson coefficients for flavor observables in the next section.

We begin by quoting the explicit formulas for the soft masses for general type I squark models (see appendix C). For concreteness, we focus on $Q$-class models, where we have (in addition to the GMSB contributions),

$$
\begin{aligned}
\delta m_{Q}^{2} & =\frac{d_{Q}}{256 \pi^{4}}\left(\left(d_{\phi}+d_{Q}\right) \kappa^{2}-2 C_{r} g_{r}^{2}-\frac{16 \pi^{2}}{3} h\left(\frac{\Lambda}{M}\right) \frac{\Lambda^{2}}{M^{2}}\right) K \Lambda^{2} \\
\delta m_{U}^{2} & =-\frac{d_{Q} d_{U}^{Q H}}{256 \pi^{4}} y_{u} K y_{u}^{\dagger} \Lambda^{2} \\
\delta m_{H_{u}}^{2} & =-\frac{3 d_{Q}}{256 \pi^{4}} \operatorname{Tr}\left[y_{u} K y_{u}^{\dagger}\right] \Lambda^{2} \\
A_{\tilde{u}} & =-\frac{d_{Q} \Lambda}{16 \pi^{2}} y_{u} K .
\end{aligned}
$$


Here we are neglecting the (numerically irrelevant) down-Yukawa contributions; $K$ is the rank one matrix of couplings defined in (1.4); $d_{U}^{Q H}=2$ is a multiplicity factor; and $h(x)$ is an $\mathcal{O}(1)$ loop function (see (C.8) for the exact form). For our $Q$-class model of study (1.2), we have $d_{Q}=N=6, d_{\phi}=5$, where $N$ is the number of messengers. ${ }^{2}$

We can see the $\chi \mathrm{FV}$ texture of (2.3) quite clearly in the formulas for $\delta m_{U}^{2}$ and $A_{\tilde{u}}$ in (2.6). There are also additional features of these explicit formulas that go beyond the $\chi \mathrm{FV}$ ansatz. We notice that $\delta m_{Q}^{2}$, together with the quantities $\Gamma$ and $\Sigma$ introduced in (2.2), are all proportional to $K$ :

$$
\delta m_{Q}^{2}=\beta K, \quad \Gamma=\gamma K, \quad \Sigma=\sigma K
$$

The simplicity of these relations is partly due to the rank 1 nature of the flavor violation. But in principle, on symmetry grounds alone, there could have been additional contributions to (2.7) proportional to the identity matrix and powers of the Yukawa couplings. These are absent due to the specific form of the type I squark couplings. This is the main reason we have focused on the type I models in this paper. The forms of the soft masses are more complicated in the type II models, and while we expect them to be similarly protected by their $\chi \mathrm{FV}$ flavor texture, understanding them at the level of analytical detail that we apply to the type I models is more difficult.

Using the relations (2.7), we now discuss the diagonalization of the squark mass matrices. Because the LR blocks are suppressed by $v / m_{\mathrm{SUSY}}$, the squark mass eigenvalues are given by those of the LL and RR blocks to a very good approximation. The RR blocks are already diagonal in the third-generation dominant approximation according to (2.3). The down-squark RR masses are just their minimal GMSB values $m_{0}^{2}$, while the up-squark RR masses are given by $m_{0}^{2}, m_{0}^{2}$ and $m_{0}^{2}+\sigma y_{t}^{2} \kappa_{3}^{2} \equiv m_{R R}^{2}$. In practice the third eigenvalue is only a little offset from $m_{0}^{2}$ throughout the parameter space of our model, and this difference can be neglected.

The situation for the LL block is only a little more complicated. Since $\delta m_{Q}^{2}$ is just proportional to $K$, the LL block of the squark mass matrices is diagonalized by the unitary transformation

$$
U_{L L}=\left(\begin{array}{lll}
\hat{\kappa}_{1} & a_{1} & b_{1} \\
\hat{\kappa}_{2} & a_{2} & b_{2} \\
\hat{\kappa}_{3} & a_{3} & b_{3}
\end{array}\right)
$$

where $\hat{\kappa}_{a}=\kappa_{a} / \kappa$ and $\vec{a}$ and $\vec{b}$ are any two orthonormal basis vectors for the orthogonal subspace $\vec{\kappa}^{\perp}$. The eigenvalues are $m_{0}^{2}, m_{0}^{2}$, and

$$
m_{S}^{2} \equiv m_{0}^{2}+\beta \kappa^{2}
$$

(Here we are ignoring the small differences between the minimal GMSB contributions to the $Q, U, D$ soft masses coming from $\mathrm{SU}(2) \times \mathrm{U}(1)$.) In our EGMSB models, $m_{S}^{2}$ corresponds roughly to the mass of the lightest squark, and moreover

$$
m_{S}^{2} \ll m_{0}^{2}
$$

\footnotetext{
${ }^{2}$ For the similar $U$-class model formulas, simply change $U \leftrightarrow Q, A_{u} \rightarrow A_{u}^{\dagger}, y_{q} \rightarrow y_{q}^{\dagger}$, and $K \rightarrow K^{T}$. The analogous multiplicity factor is $d_{Q}^{U H}=1$, and for our $U$-class model, we have $d_{U}=2 N=6, d_{\phi}=4$.
} 
Thus the Wilson coefficients will generally be dominated by this lightest squark running in the loop, and $m_{S}^{2}$ will play a central role in controlling the size of the flavor-violating effects in these models. In fact, because of (2.10), diagrams where the right-handed squarks propagate tend to be suppressed, and we will see that it suffices to focus on the LL flavor violation exclusively.

\section{Flavor observables and constraints on EGMSB}

In this section, we investigate the flavor constraints on our EGMSB models (1.2) in detail. For reference, the spectra corresponding to $\kappa_{1}=\kappa_{2}=0$ are shown in figure 1 . These are essentially the same points that were identified in [20] as being the least fine-tuned EGMSB models with $m_{h}=125 \mathrm{GeV}$. Starting from these flavor-aligned points, we will perform a numerical scan in the flavor-violating parameter space $\left(\kappa_{1} / \kappa_{3}, \kappa_{2} / \kappa_{3}\right)$ of the models, using FormFlavor to compute the flavor observables and compare against their experimental values. Going through each observable $X$ from table 1 in turn, we will exhibit plots of

$$
\frac{\left|[X]_{\mathrm{TH}}-[X]_{\mathrm{EXP}}\right|}{[\sigma(X)]_{\mathrm{TH}+\mathrm{EXP}}}
$$

Here $[X]_{\mathrm{TH}}$ denotes the SM prediction together with the EGMSB contribution, and $[\sigma(X)]_{\mathrm{TH}+\mathrm{EXP}}$ is the theoretical and experimental errors added in quadrature. A contour of 2 , which is roughly the $95 \%$ CL exclusion limit, will be taken to indicate the point at which the EGMSB model is excluded by the given flavor observable. ${ }^{3}$

$$
\frac{\left|\left[\Delta m_{X}\right]_{\mathrm{EGMSB}}\right|}{\left[\Delta m_{X}+2 \sigma\left(\Delta m_{X}\right)\right]_{\mathrm{EXP}}}
$$

Thus a contour of 2 does not represent a $95 \%$ confidence level exclusion for these observables. However, as it would require a substantial tuning for the standard model and the new physics contributions to cancel against one another, values larger than 1 are suspect.

As we deform away from $\kappa_{1}=\kappa_{2}=0$, there are numerous subtleties that must be taken into account regarding how the other parameters of the model are varied. These subtleties and specifics of the procedure are described in appendix A. In short, $\kappa_{1}$ and $\kappa_{2}$ are introduced in such a way that the superpartner mass eigenvalues and "net" $A$-terms are essentially held fixed.

In order to validate the numerical results from FormFlavor, we will compare them against analytical formulas for the flavor observables. This will also shed further qualitative insights on the role of $\chi \mathrm{FV}$ in weakening the flavor constraints. As discussed in the Introduction, the usual mass insertion approximation fails due to $\mathcal{O}(1)$ entries. Instead, we will use flavor symmetries and the special features of the type I models discussed above to characterize their exact $\kappa$ dependence, to leading order in an expansion in $v / m_{S}$. In general, the $\kappa$ dependence arises through the lightest squark mass (2.9) and the unitary matrix $U_{L L}$ given in (2.8). (The LR and RL blocks in (2.3) also depend on $\kappa$ through (2.7),

\footnotetext{
${ }^{3}$ Two exceptions to this are $\Delta m_{K}$ and $\Delta m_{D}$ where the theory errors are uncontrolled. In these cases, we will plot instead:
} 

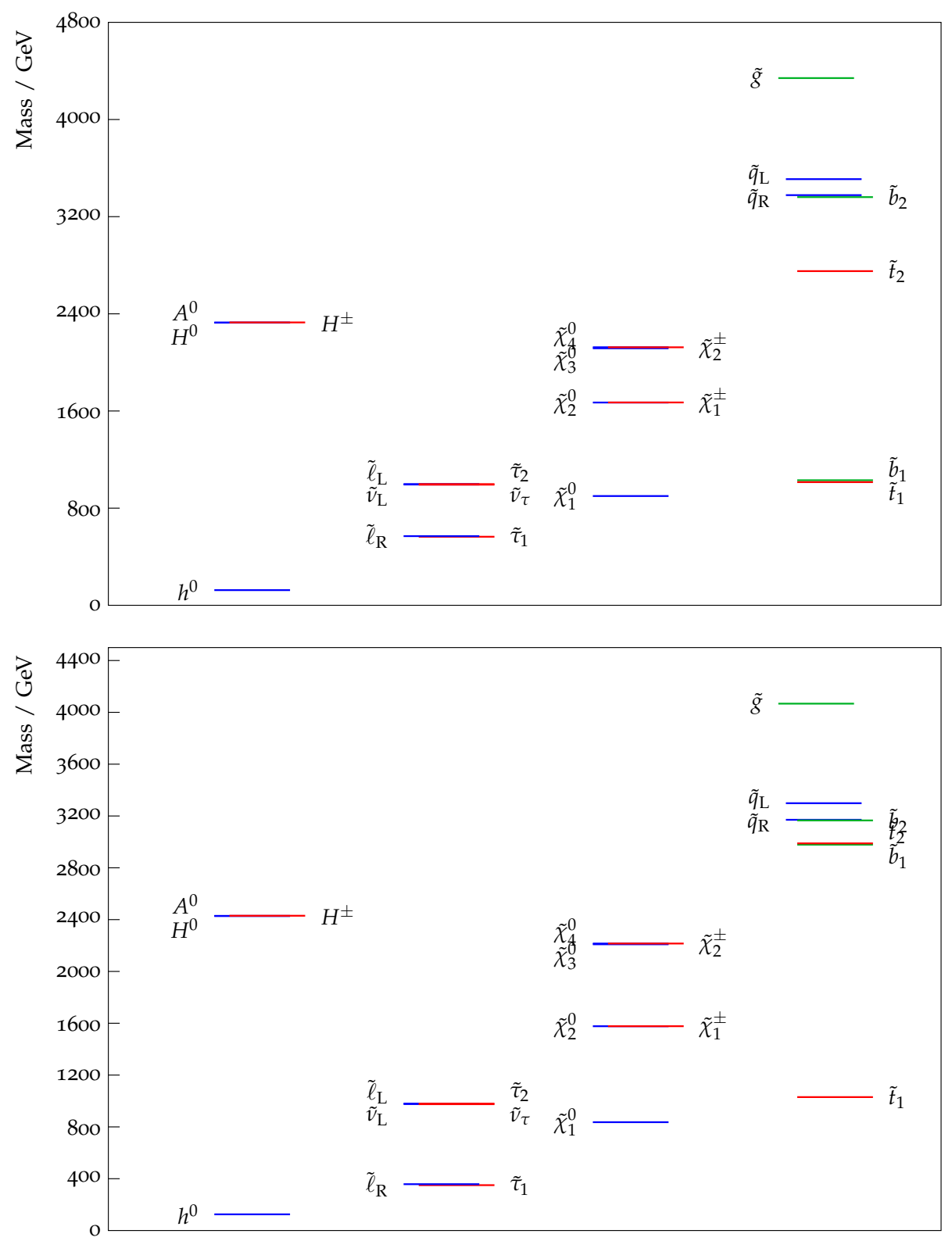

Figure 1. Top: mass spectrum of the $\kappa_{i} Q_{i} \Phi_{D} \Phi_{L}$ model at $\kappa_{1}=\kappa_{2}=0$. Bottom: mass spectrum of the $\kappa_{i} U_{i} \Phi_{D_{1}} \Phi_{D_{2}}$ model at $\kappa_{1}=\kappa_{2}=0$.

but as discussed in the previous section, this dependence can be generally be neglected due to the heaviness of the right-handed squarks and the extra $v / m_{S}$ suppression.) The dependence on $U_{L L}$ is constrained by the flavor symmetries, and as explained in the previous paragraph, the squark mass eigenvalues are mostly held fixed in our parameter space. Thus it will be possible to fully characterize the features of the FormFlavor plots in terms of very simple functions of $\kappa$. 


\subsection{Q-class models}

\subsubsection{Meson mixing}

We begin with the $\Delta F=2$ meson mixing observables. As we discuss in appendix D.1, for $X=K$ and $D$,

$$
\Delta m_{X}=2 \operatorname{Re}\left\langle\bar{X}\left|H_{\text {eff }}\right| X\right\rangle
$$

to a good approximation, while for $X=B_{d}$ and $B_{q}$,

$$
\Delta m_{X}=2\left|\left\langle\bar{X}\left|H_{\text {eff }}\right| X\right\rangle\right|
$$

to a good approximation. Here $H_{\text {eff }}$ is the $\Delta F=2$ effective Hamiltonian; its local shortdistance part (relevant for the MSSM contributions) is built out of the four-fermi operators,

$$
\begin{aligned}
\left(\mathcal{O}_{S}^{M N}\right)_{a b} & =\left(\bar{q}_{a} P_{M} q_{b}\right)\left(\bar{q}_{a} P_{N} q_{b}\right) \\
\left(\mathcal{O}_{V}^{M N}\right)_{a b} & =\left(\bar{q}_{a} \gamma^{\mu} P_{M} q_{b}\right)\left(\bar{q}_{a} \gamma_{\mu} P_{N} q_{b}\right) \\
\left(\mathcal{O}_{T}^{M N}\right)_{a b} & =\left(\bar{q}_{a} \sigma^{\mu \nu} P_{M} q_{b}\right)\left(\bar{q}_{a} \sigma_{\mu \nu} P_{N} q_{b}\right)
\end{aligned}
$$

Here $M, N=L, R$ label the chirality of the incoming quarks; and $a b=12,12,13,23$ for $\Delta m_{K}, \Delta m_{D}, \Delta m_{B_{d}}$ and $\Delta m_{B_{s}}$, respectively, while $q$ is up-type for $\Delta m_{D}$ and down-type for the rest.

The full result of FormFlavor is shown in figure 2. In this subsection, we will endeavor to understand its features analytically using the $\chi \mathrm{FV}$ ansatz and the special features of type I EGMSB identified in section 2.2.

The MSSM contributions to the $\Delta F=2$ observables are due to box diagrams involving the squarks and the gauginos. Here the great simplification of $\chi \mathrm{FV}$ is that any operator with an $R$ index must transform non-trivially under $\mathrm{SU}(3)_{D}$ or $\mathrm{SU}(3)_{U}$; thus it is suppressed by $\chi \mathrm{FV}$ in our $Q$-class models. Furthermore, the $\mathcal{O}_{S}^{L L}$ and $\mathcal{O}_{T}^{L L}$ operators all involve an $\mathrm{SU}(2)_{L}$-breaking chirality flip, so they are dropped in the $v=0$ approximation. Therefore, the only unsuppressed Wilson coefficient is $C_{V}^{L L}$. This also happens to be the only contribution to the one-loop SM Wilson coefficients, which proceeds through $W$ exchange.

Since $C_{V}^{L L}$ transforms in the square of the adjoint+singlet representation of $\mathrm{SU}(3)_{Q}$, with just left-handed squarks running in the loop, the only way it can depend on $\kappa$ is,

$$
\left(C_{V}^{L L}\right)_{a b}=\frac{\hat{\kappa}_{a}^{2} \hat{\kappa}_{b}^{2}}{m_{S}^{2}} f_{1}\left(m_{S}^{2} / m_{0}^{2}\right)
$$

where $f_{1}$ is a dimensionless loop function depending on the LL squark mass eigenvalues. ${ }^{4}$ Dimensional analysis fixes the dependence on the masses, and the rest of the dependence must be from the unitary matrix (2.8). Under this simplification, the meson mixing contributions are of the form (see appendix D.1 for more details and specific values of the parameters),

$$
\left[\left\langle\bar{X}\left|H_{\mathrm{eff}}\right| X\right\rangle\right]_{\mathrm{EGMSB}} \approx \frac{1}{3} m_{X} f_{X}^{2} B_{V, X}^{L L}\left(C_{V}^{L L}\right)_{a b},
$$

\footnotetext{
${ }^{4}$ All loop functions here and below will implicitly depend on the masses of the other superpartners running in the loop, e.g., the gaugino masses.
} 

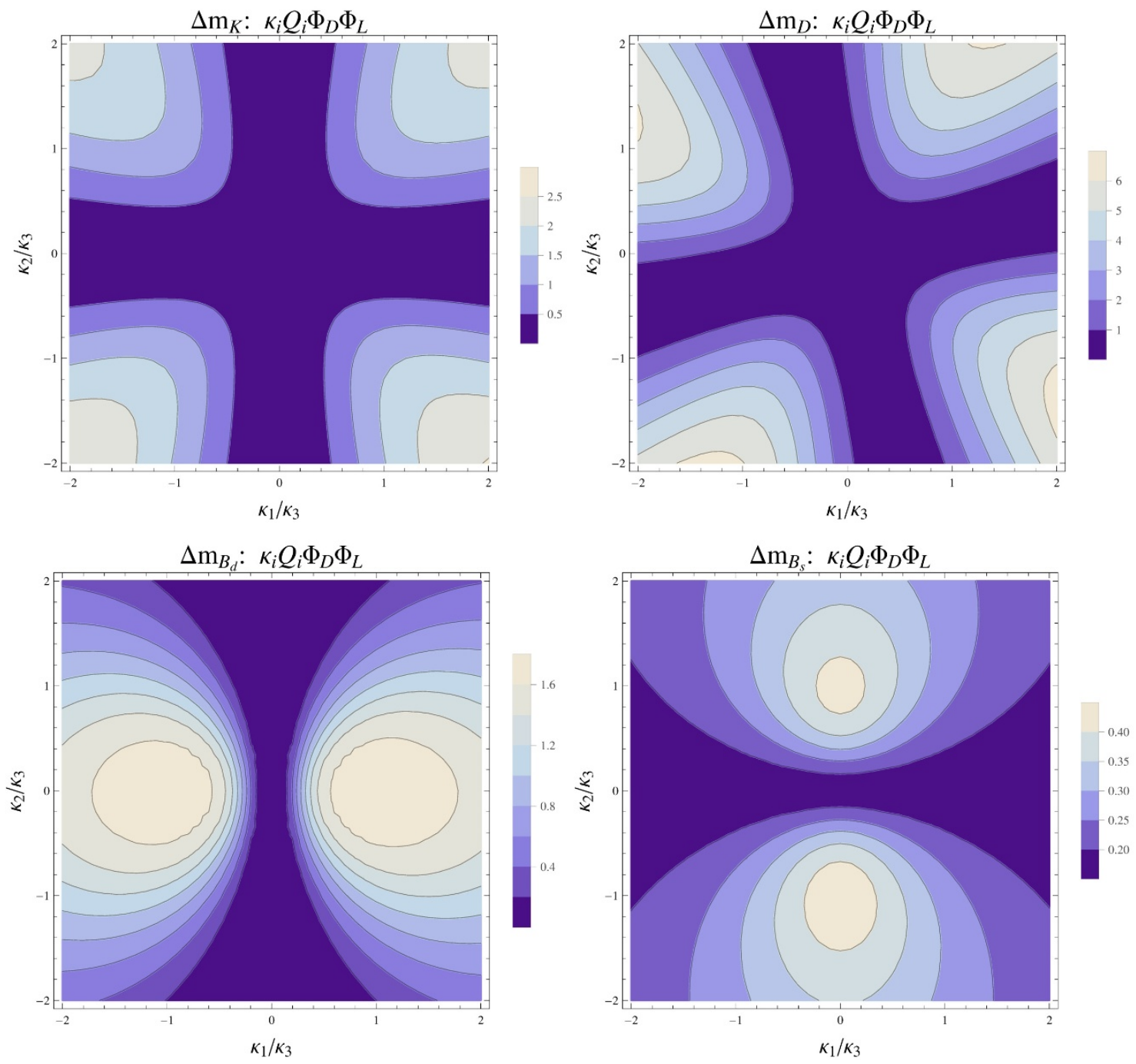

Figure 2. Plots of meson mixing observables the $Q$-class model, $\kappa_{i} Q_{i} \Phi_{D} \Phi_{L} . \Delta m_{K}, \Delta m_{D}, \Delta m_{B_{d}}$, $\Delta m_{B_{s}}$ are presented in the upper left, upper right, lower left and lower right, respectively. Both $\Delta m_{K}$ and $\Delta m_{D}$ are presented as the FormFlavor output over the experimental value, while $\Delta m_{B_{d}}$ and $\Delta m_{B_{s}}$ are the difference between FormFlavor and the experimental value in units of the uncertainty.

where $f_{X}$ is the decay constant for the meson; and $B_{V, X}^{L L}$ is an $\mathcal{O}(1)$ hadronic parameter. This simple formula suffices to accurately describe the $\Delta F=2$ flavor observables.

Focusing on gluino boxes for simplicity ${ }^{5}$ we find from explicit computation that the leading Wilson coefficient is given by

$$
\left(C_{V}^{L L}\right)_{a b}=-\hat{\kappa}_{a}^{2} \hat{\kappa}_{b}^{2} \frac{\alpha_{s}^{2}}{36 m_{S}^{2}} f_{\tilde{g}}^{\Delta M, \text { box }}\left(x_{g}, x_{q}\right)
$$

\footnotetext{
${ }^{5}$ Chargino and gluino-neutralino boxes are also typically comparable. However, they do not affect the qualitative discussion here. In fact, because the charginos enter with the opposite sign of the gluino and gluino+neutralino contributions, the gluinos alone provide a better estimate quantitatively than might be expected.
} 
This clearly agrees with the general form (3.6). Here, $x_{q}=m_{S}^{2} / m_{0}^{2}$ and $x_{g}=m_{\tilde{g}}^{2} / m_{0}^{2}$, and $f_{\tilde{g}}^{\Delta M \text {,box }}\left(x_{g}, x_{q}\right) \approx 0.05$ is a loop function that we define in appendix E. As discussed earlier, since the relevant masses are roughly held fixed in our deformation, the loop function does not change across the parameter space. The QCD RG running from the SUSY scale to the meson scale is fairly mild for $C_{V}^{L L}$, yielding only a $20-30 \%$ suppression in the size of the Wilson coefficient [52].

The hadronic factors $f_{X}^{2} B_{V, X}^{L L}$ are fairly similar across the mesons. We can define,

$$
H_{X} \equiv \frac{f_{X}^{2} B_{V, X}^{L L}}{f_{K}^{2} B_{V, K}^{L L}} ; \quad H_{K}=1, H_{D} \approx H_{B_{d}} \approx 2, H_{B_{s}} \approx 3
$$

So we expect deviations of the form,

$$
\left[\left\langle\bar{X}\left|H_{\mathrm{eff}}\right| X\right\rangle\right]_{\mathrm{EGMSB}} \sim-\hat{\kappa}_{a}^{2} \hat{\kappa}_{b}^{2} H_{X} m_{X}\left(10^{-13}\right),
$$

Noting that $\hat{\kappa}_{a}^{2} \hat{\kappa}_{b}^{2}$ is at most $\frac{1}{4}$, and comparing against table 1 , we see that $\Delta m_{K}$ and $\Delta m_{D}$ should be most sensitive to EGMSB, while $\Delta m_{B_{d}}$ should be barely sensitive, and $\Delta m_{B_{s}}$ completely insensitive. ${ }^{6}$

These sensitivities are observed in the plots shown in figure 2. Moreover, the $\hat{\kappa}_{a}^{2} \hat{\kappa}_{b}^{2}$ dependence is transparent in these plots. $\Delta B_{d}$ peaks at $\left(\kappa_{1} / \kappa_{3}, \kappa_{2} / \kappa_{3}\right) \sim( \pm 1,0)$, while $\Delta B_{s}$ peaks at $\sim(0, \pm 1)$. For $\Delta m_{K}$ and $\Delta m_{D}$, we can see that moving from $\kappa_{1}=\kappa_{2}=\kappa_{3}$ to the corners, constraints rise by the expected factor of $\sim 16 / 9 .{ }^{7}$

These results from meson mixing may seem to conflict with the SUSY flavor problem, which suggests that, with $\mathcal{O}(1)$ flavor-violation, the SUSY scale needs to enter above $\sim 500 \mathrm{TeV}$ due to constraints from $\Delta m_{K}$ and $\Delta m_{D}[49,50]$. However, these constraints are driven by the $C_{S}^{L R}$ Wilson coefficient, while $\chi \mathrm{FV}$ only generates the $C_{V}^{L L}$ operator. In the MSSM, the contribution of the latter to $\Delta m_{K, D}$ is suppressed by $\sim 10^{-4}-10^{-3}$ relative to the former. This is due to three separate effects that all work in the same direction. First, the hadronic matrix elements differ between these two operators,

$$
\frac{\left\langle K\left|S_{L R}\right| \bar{K}\right\rangle}{\left\langle K\left|V_{L L}\right| \bar{K}\right\rangle}=\frac{3}{4} \frac{B_{S}^{L R}}{B_{V}^{L L}} R_{K} \sim 35, \quad \frac{\left\langle D\left|S_{L R}\right| \bar{D}\right\rangle}{\left\langle D\left|V_{L L}\right| \bar{D}\right\rangle}=\frac{3}{4} \frac{B_{S}^{L R}}{B_{V}^{L L}} R_{D} \sim 4 .
$$

Next, the SUSY contributions to the Wilson coefficients are also quite different in size. From the MIA with $\mathcal{O}(1)$ mass-insertions, it is easy to see that $C_{S}^{L R} / C_{V}^{L L} \sim 30$ [49]. Lastly, the QCD running from the SUSY scale to $\sim 2 \mathrm{GeV}$ suppresses $C_{V}^{L L}$ by $20-30 \%$, but enhances $C_{S}^{L R}$ by a factor of $\sim 3$ [52]. All of these factors conspire to drop the scale of sensitivity to $\Delta m_{K}$ and $\Delta m_{D}$ to the TeV scale in $\chi \mathrm{FV}$ models.

\footnotetext{
${ }^{6}$ As discussed in the Introduction, we are not considering CP violation in this work, in particular $\epsilon_{K}$. Although it depends on the precise value of the $\mathrm{CP}$ violating phase, the expectation is that this will place a meaningful, tighter constraint on the parameter space when the phase is large. This and other CPV observables will be studied in an upcoming paper [35].

${ }^{7}$ Note that $\Delta m_{D}$ is rotated by the Cabbibo angle $\theta_{c}$ relative to the other observables. This is a consequence of the the fact that, as discussed in in appendix A, our $\kappa_{1}, \kappa_{2}$, and $\kappa_{3}$ directions are chosen to align with the low energy down, strange and bottom quark. For $\Delta m_{D}$, the dependence on $\kappa_{i}$ proceeds through the LL sector of the up-squark mass matrix, where there is an additional rotation by $V_{C K M}$.
} 


\subsection{2 $K^{ \pm} \rightarrow \pi^{ \pm} \nu \bar{\nu}$}

Unlike meson mixing in the previous subsection, the $K^{ \pm} \rightarrow \pi^{ \pm} \nu \bar{\nu}$ observable (along with all other $\Delta F=1$ observables) enters as a matrix element squared, and thus interference with the standard model contribution can be important. The expression for this branching ratio is (see appendix D.2),

$$
\mathrm{BR}\left(K^{ \pm} \rightarrow \pi^{ \pm} \nu \bar{\nu}\right)=c_{+} v^{4}\left|C_{V, \mathrm{SM}}^{L L}+C_{V}^{L L}+C_{V}^{R L}\right|^{2}
$$

where $c_{+} v^{4}=4.9 \times 10^{9} \mathrm{GeV}^{4}, C_{V, \mathrm{SM}}^{L L}=(-1.21+0.39 i) \times 10^{-10} \mathrm{GeV}^{-2}$, and all EGMSB effects are contained in $C_{V}^{L L}$ and $C_{V}^{R L}$. These are the Wilson coefficients from the $\Delta F=1$ effective Hamiltonian built out of the four-fermi operators

$$
\begin{aligned}
\left(\mathcal{O}_{S}^{M N}\right)_{a b} & =\left(\bar{q}_{a} P_{M} q_{b}\right)\left(\bar{\ell} P_{N} \ell\right) \\
\left(\mathcal{O}_{V}^{M N}\right)_{a b} & =\left(\bar{q}_{a} \gamma^{\mu} P_{M} q_{b}\right)\left(\bar{\ell} \gamma_{\mu} P_{N} \ell\right) \\
\left(\mathcal{O}_{T}^{M N}\right)_{a b} & =\left(\bar{q}_{a} \sigma^{\mu \nu} P_{M} q_{b}\right)\left(\bar{\ell} \sigma_{\mu \nu} P_{N} \ell\right)
\end{aligned}
$$

Here $a b=12$ for $K^{ \pm} \rightarrow \pi^{ \pm} \nu \bar{\nu}$. In the MSSM, these Wilson coefficients arise through one-loop box and $Z$-penguin diagrams.

The full FormFlavor result is shown in figure 3. The general trend of $K^{ \pm} \rightarrow \pi^{ \pm} \nu \bar{\nu}$ can again be understood through use of the features of rank $1 \chi \mathrm{FV}$ discussed in section 2.2. First of all, as for the $\Delta F=2$ observables, $C_{V, a b}^{R L}$ must be zero in the third-generation dominant approximation - since it transforms in the adjoint+singlet of $\mathrm{SU}(3)_{D}$, using the available spurions one can only obtain something that is nonzero in the 33 component. Thus, we can focus on $C_{V, a b}^{L L}$. This transforms in the adjoint+singlet of $\mathrm{SU}(3)_{Q}$. Using flavor violation in the LL block only, the form of $C_{V, a b}^{L L}$ is constrained by the symmetries to be:

$$
C_{V, a b}^{L L}=\frac{\hat{\kappa}_{a} \hat{\kappa}_{b}}{m_{S}^{2}} f_{2}+\ldots
$$

where $\ldots$ contains higher orders in $v / m_{\text {SUSY }}$ and other irrelevant terms, and $f_{2}$ is a dimensionless function of superpartner mass ratios. We note that (3.14) can come from box diagrams or $Z$-penguin diagrams, but in the latter case, the $1 / m_{Z}^{2}$ from the $Z$ propagator must be canceled out by two insertions of wino-Higgsino mixing. Insertions of LR mixing from the squark mass matrix would also cancel out the $1 / m_{Z}^{2}$, but the heavy right-handed squark masses suppress these contributions enough that they may be ignored.

Now we will compare against explicit computations of the Wilson coefficients. We confirm that the $C_{V}^{R L}$ coefficients are all negligible. For $C_{V}^{L L}$, we find that the chargino diagrams dominate, and they are given by

$$
C_{V}^{L L}=\hat{\kappa}_{1} \hat{\kappa}_{2} \frac{\alpha_{2}^{2}}{12 m_{S}^{2}} f_{\tilde{\chi}^{ \pm}}^{K \rightarrow \pi \nu \nu}\left(x_{l}, x_{2}, x_{\mu}\right)
$$

Again, $f_{\tilde{\chi}^{ \pm}}^{K \rightarrow \pi \nu \nu}\left(x_{\ell}, x_{2}, x_{\mu}\right) \sim 1.5$ is a loop function of the sparticle mass-ratios $x_{\ell}=m_{\tilde{\ell}}^{2} / m_{S}^{2}$, $x_{2}=M_{2}^{2} / m_{S}^{2}$, and $x_{\mu}=\mu^{2} / m_{S}^{2}$, defined in appendix E, and varies only mildly across the parameter space. This is fully consistent with the general form (3.14). This also explains 


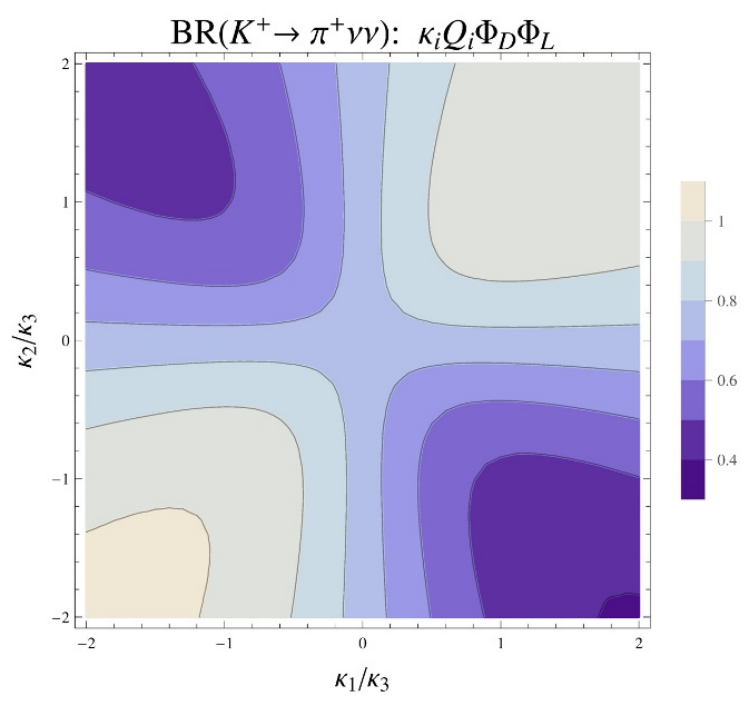

Figure 3. Contours of the difference in $\mathrm{BR}\left(K^{+} \rightarrow \pi^{+} \nu \bar{\nu}\right)$ between experiment and the $\mathrm{SM}+$ EGMSB predictions, given in units of the net uncertainty (added in quadrature). A contour of 2 corresponds roughly to a $95 \%$ exclusion.

why the charginos dominate over the gluinos, since the gluinos can only give rise to $Z$ penguin diagrams that are nonzero by virtue of down sector LR squark-mixing insertions.

Substituting in numerically for the loop function, $m_{S}$ and $\alpha_{2}$, we find

$$
C_{V}^{L L} \sim\left(8 \times 10^{-11} \mathrm{GeV}^{-2}\right) \hat{\kappa}_{1} \hat{\kappa}_{2}
$$

This simple function of $\vec{k}$, when added to the SM contribution and substituted into (3.12), reproduces well the features of figure 3 . It grows in magnitude fastest along the lines $\kappa_{1}= \pm \kappa_{2}$, asymptoting to the values $\pm 4 \times 10^{-11} \mathrm{GeV}^{-2}$. In the corners of the parameter space we obtain a deviation from the SM prediction of

$$
\Delta \operatorname{BR}\left(K^{+} \rightarrow \pi^{+} \nu \bar{\nu}\right)=c_{+} v^{4}\left(2 \operatorname{Re}\left[C_{V, \mathrm{SM}}^{L L} C_{V}^{L L *}\right]+\left|C_{V}^{L L}\right|^{2}\right) \approx \mp 5 \times 10^{-11},
$$

This is smaller than the current experimental+theoretical uncertainty shown in table 1 . However, because the SM prediction for the BR is a little lower than the experimentally observed value, moving along the $\kappa_{1}=+\kappa_{2}$ line slightly exacerbates the difference, while moving along the $\kappa_{1}=-\kappa_{2}$ line slightly lessens it.

\subsection{3 $\quad b \rightarrow s \gamma$ and $b \rightarrow d \gamma$}

The expression for the $b \rightarrow s \gamma$ branching ratio (see appendix D.3) is,

$$
\mathrm{BR}(b \rightarrow s \gamma)=c_{\gamma} v^{2}\left(\left|C_{A, \mathrm{SM}}^{L}+C_{A}^{L}\right|^{2}+\left|C_{A}^{R}\right|^{2}\right),
$$

where $c_{\gamma} v^{2}=1.97 \times 10^{12} \mathrm{GeV}^{2}$ and $C_{A, \mathrm{SM}}^{L} \sim 1.3 \times 10^{-8} \mathrm{GeV}^{-1}$. The new physics is contained in $C_{A}^{L}$ and $C_{A}^{R}$; these come from the effective Hamiltonian built out of the dimension 5 operators

$$
\left(\mathcal{O}_{A}^{M}\right)_{a b}=e\left(\bar{q}_{a} \sigma^{\mu \nu} P_{M} q_{b}\right) F_{\mu \nu}
$$



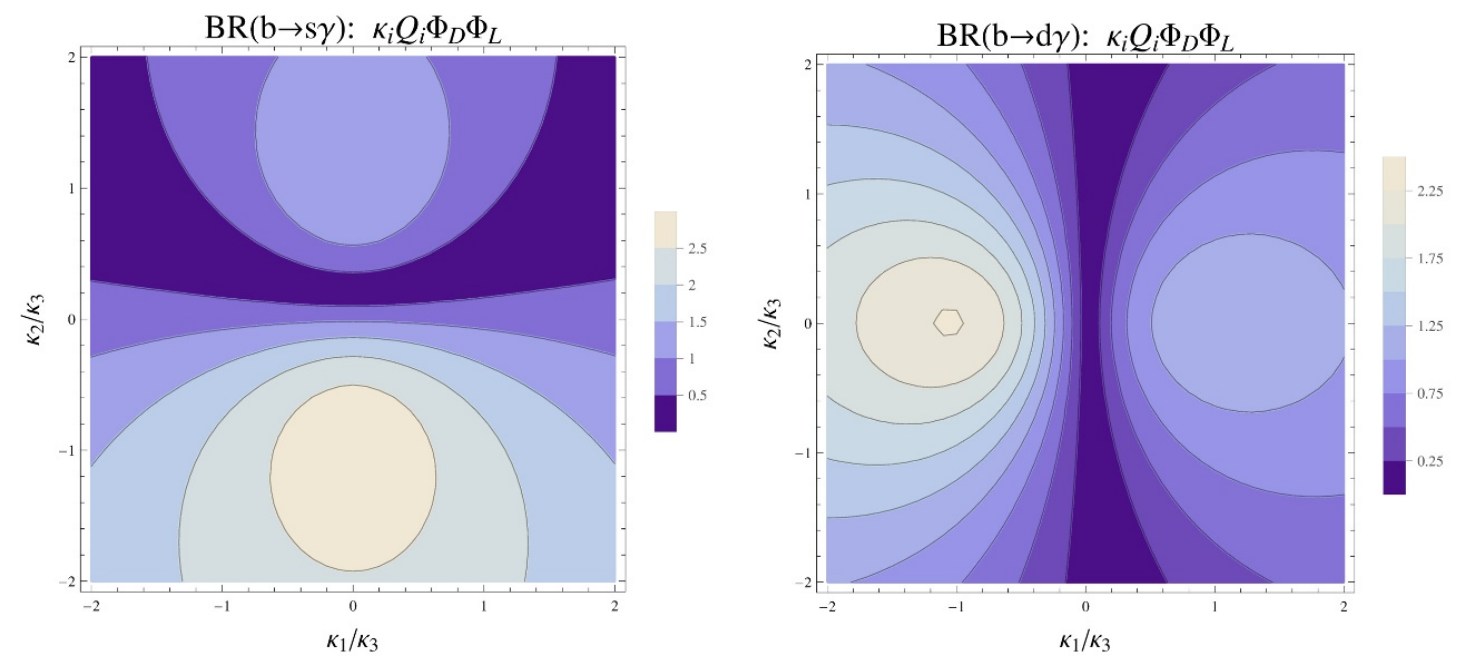

Figure 4. Sensitivity to $b \rightarrow s \gamma$ (left) and $b \rightarrow d \gamma$ (right), same conventions as figure 3 .

where $a b=32$ for $b \rightarrow s \gamma$. These operators require $\mathrm{SU}(2)$ breaking, so their Wilson coefficients are zero in the $v \rightarrow 0$ limit. Naively, this would mean that the result is negligible in our zeroth-order expansion in $v / m_{\text {SUSY }}$. However, the SM Wilson coefficient is suppressed by $m_{b} / v$, so here we consider one higher order in the $v / m_{\mathrm{SUSY}}$ expansion in order to capture a numerically relevant result.

Since $C_{A}^{L}\left(C_{A}^{R}\right)$ transforms in the $(\mathbf{3}, \overline{\mathbf{3}})$ of $\mathrm{SU}(3)_{D} \times \mathrm{SU}(3)_{Q}\left(\mathrm{SU}(3)_{Q} \times \mathrm{SU}(3)_{D}\right)$, it must be proportional to $m_{d}$ acting on the left (right). The latter is zero in the thirdgeneration dominant approximation, so it suffices to focus on $C_{A}^{L}$. With just left-handed squarks propagating in the loop, the Wilson coefficient must be given by:

$$
C_{A, 32}^{L}=\frac{\left(m_{d} K \tan \beta\right)_{32}}{\kappa^{2}} \frac{1}{m_{S}^{2}} f_{6}=\hat{\kappa}_{2} \hat{\kappa}_{3} \frac{m_{b} \tan \beta}{m_{S}^{2}} f_{6}
$$

Here we assumed that $m_{d}$ is accompanied by a $\tan \beta$ enhancement, otherwise the entire effect is numerically negligible.

Comparing with explicit calculation, we find again that charginos give the dominant contribution to the Wilson coefficient, through the quark-squark-Higgsino vertex. (Contributions of the form (3.20) can also arise through gluino and neutralino loops, but here the factor of $m_{b} \tan \beta$ arises through the LR block of the down-squark mass matrix, so the diagrams are suppressed by heavy right-handed squarks propagating in the loop.) The dominant chargino diagram gives,

$$
C_{A}^{L}=\hat{\kappa}_{2} \hat{\kappa}_{3} \frac{m_{b} \tan \beta}{m_{S}^{2}} \frac{11 \alpha_{2}}{288 \pi} f_{\tilde{\chi}^{ \pm}}^{b \rightarrow s / d \gamma, \text { peng }}\left(x_{\mu}, x_{2}\right),
$$

where $x_{\mu}=\mu^{2} / m_{S}^{2}, x_{2}=M_{2}^{2} / m_{S}^{2}$, and $f_{\tilde{\chi}^{ \pm}}^{b \rightarrow s / d \gamma \text {,peng }}\left(x_{\mu}, x_{2}\right) \sim 0.5$ is a loop function defined in appendix E. This is fully in agreement with the general result (3.20). QCD running from the SUSY scale to the $b$ pole [53] induces a mild $20 \%$ suppression to (3.21), and once again, we find this simple result is enough to account for the features of the FormFlavor plot in figure 4 . 
Substituting in numerically for our parameter space (we take $\tan \beta=10$ as in [20]), we obtain:

$$
C_{A}^{L} \sim\left(3 \times 10^{-9} \mathrm{GeV}^{-1}\right) \hat{\kappa}_{2} \hat{\kappa}_{3}
$$

Clearly its effects are largest when $\kappa_{1}=0$, and along the $\kappa_{2}$ axis it has a maximum (minimum) at $\kappa_{2} / \kappa_{3}=1(-1)$, while asymptoting back to zero as $\kappa_{2} / \kappa_{3} \rightarrow \pm \infty$. Substituting this into (3.18) together with the SM Wilson coefficient, we find at these extrema a deviation from the SM prediction of

$$
[\Delta \operatorname{BR}(b \rightarrow s \gamma)]_{\kappa_{2}= \pm \kappa_{3}} \sim c_{\gamma} v^{2}\left(2 \operatorname{Re}\left[C_{A, \mathrm{SM}}^{L} C_{A}^{L *}\right]\right) \sim \pm 7 \times 10^{-5}
$$

Since the uncertainty on the measurement (combining the theoretical and experimental in quadrature) is $3.5 \times 10^{-5}$, regions of exclusion are to be expected for $b \rightarrow s \gamma$.

In practice, FormFlavor does find a region of exclusion in the bottom half of the parameter space, shown in figure 4. This exclusion is in part due to the current $\mathcal{O}(1 \sigma)$ excess in the experimental measurement relative to the theoretical prediction, ${ }^{8}$ which allows the $\sim 2 \sigma$ change to constrain a large region of negative $\kappa_{2}$ in the plot. Uncertainty on this observable is comparable in size between theory and experiment, so improvements on either side could make this observable more constraining and in a direction that other observables currently have no sensitivity.

The observable $b \rightarrow d \gamma$ could also potentially place constraints. The EGMSB contribution to the Wilson coefficient is the same as in (3.21)-(3.22), but with $\kappa_{2} \leftrightarrow \kappa_{1}$. However, the SM contribution is of a different size, $C_{A, \mathrm{SM}}^{L} \sim-(2.4+1.1 i) \times 10^{-9} \mathrm{GeV}^{-1}$. Importantly, this is not much larger than the new physics contribution, so interference is very important. This yields an approximate deviation of,

$$
\begin{gathered}
{[\Delta \operatorname{BR}(b \rightarrow d \gamma)]_{\kappa_{1}=\kappa_{3}} \sim c_{\gamma} v^{2}\left(-2\left|\operatorname{Re}\left[C_{A, \mathrm{SM}}^{L} C_{A}^{L *}\right]\right|+\left|C_{A}^{L}\right|^{2}\right) \sim-0.8 \times 10^{-5}} \\
{[\Delta \operatorname{BR}(b \rightarrow d \gamma)]_{\kappa_{1}=-\kappa_{3}} \sim c_{\gamma} v^{2}\left(2\left|\operatorname{Re}\left[C_{A, \mathrm{SM}}^{L} C_{A}^{L *}\right]\right|+\left|C_{A}^{L}\right|^{2}\right) \sim 1.6 \times 10^{-5}}
\end{gathered}
$$

while the net uncertainty is $6.3 \times 10^{-6}$. Again, a two sigma exclusion could be possible, but it would only be expected near $\frac{\kappa_{1}}{\kappa_{3}}=-1$ due to the interference acting constructively. This is borne out by figure 4 , which shows a maximum deviation of $\approx 2.3$ times the net uncertainty. Because the measurement of $b \rightarrow d \gamma$ is fairly recent, a future improvement that pushes the experimental uncertainty to the 10-15\% level would allow for $b \rightarrow d \gamma$ to place much tighter constraints on the region of constructive interference, i.e., $\kappa_{1}<0$.

\subsection{4 $\quad B_{q} \rightarrow \mu^{+} \mu^{-}$}

As shown in figure 5 , neither $B_{s} \rightarrow \mu^{+} \mu^{-}$nor $B_{d} \rightarrow \mu^{+} \mu^{-}$is at all close to constraining our EGMSB models. We include a brief discussion of these observables just for completeness sake.

\footnotetext{
${ }^{8}$ Recently, an improved theoretical prediction of $b \rightarrow s \gamma$ was released [54]. This work predicts that $\mathrm{BR}(b \rightarrow s \gamma)=(3.36 \pm 0.23) \times 10^{-4}$, which is significantly more in line with the experimental value. Unfortunately, insufficient details are provided in that work for us to modify FormFlavor to account for this prediction.
} 

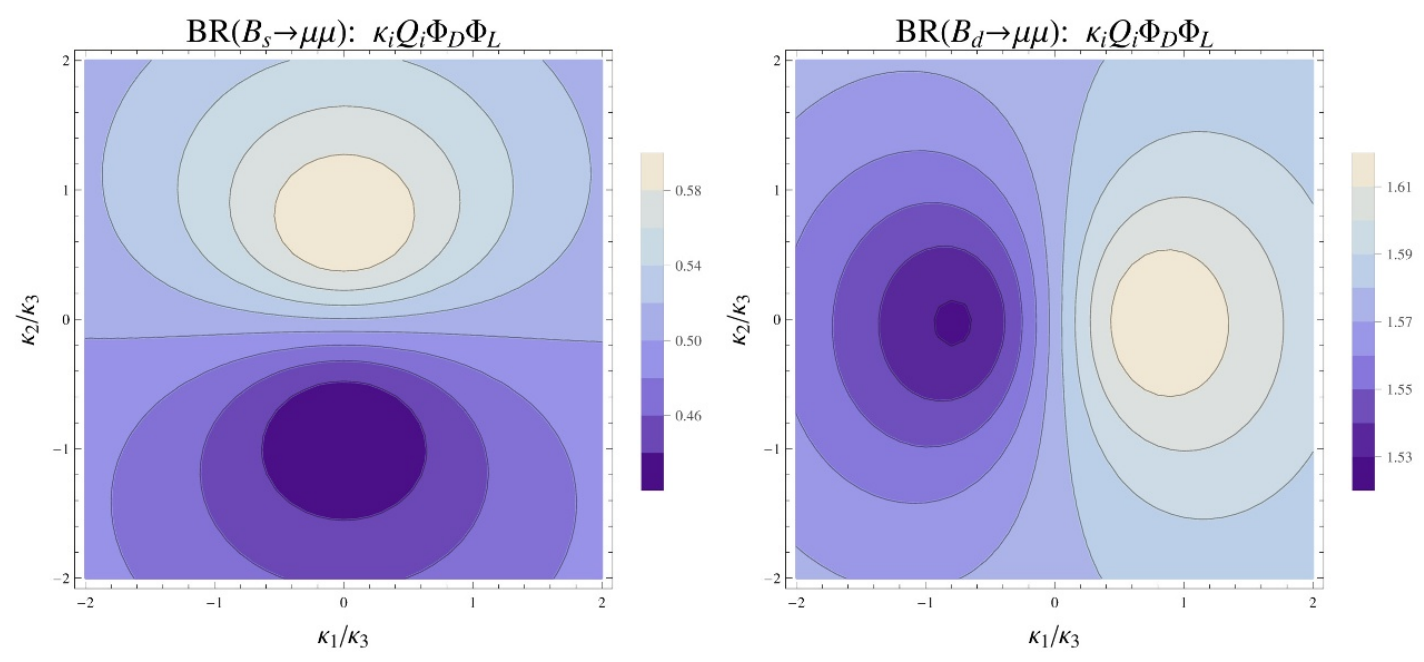

Figure 5. Sensitivity to $B_{s} \rightarrow \mu \mu$ and $B_{d} \rightarrow \mu \mu$, same conventions as figure 3. Neither is constraining over the parameter space.

The branching ratio for $B_{q} \rightarrow \mu^{+} \mu^{-}$is $[55,56]$ (see appendix D.4 for more detailed formulas):

$$
\operatorname{BR}\left(B_{q} \rightarrow \mu^{+} \mu^{-}\right)=X_{q}\left\{\left(1-\frac{4 m_{\mu}^{2}}{m_{B_{q}}^{2}}\right)\left|F_{S}^{(q)}\right|^{2}+\left|F_{P}^{(q)}+F_{A}^{(q)}\right|^{2}\right\}
$$

where $X_{s}=5.36 \times 10^{7}$ and $X_{d}=3.97 \times 10^{7}$, and

$$
\begin{aligned}
& F_{S}^{(q)}=\frac{m_{B_{q}}^{3}}{m_{b}+m_{q}}\left(C_{S}^{L L}+C_{S}^{L R}-C_{S}^{R R}-C_{S}^{R L}\right), \\
& F_{P}^{(q)}=\frac{m_{B_{q}}^{3}}{m_{b}+m_{q}}\left(-C_{S}^{L L}+C_{S}^{L R}-C_{S}^{R R}+C_{S}^{R L}\right), \\
& F_{A}^{(q)}=2 m_{B_{q}} m_{\mu}\left(C_{V}^{L L}-C_{V}^{L R}+C_{V}^{R R}-C_{V}^{R L}\right),
\end{aligned}
$$

Here the $C_{Y}^{M N}$ coefficients are those for the $\Delta F=1$ effective Hamiltonian introduced in (3.13). The three-loop Standard Model contribution [57] is $F_{A, \mathrm{SM}}^{(d)}=(1.5-0.6 i) \times 10^{-9}$ and $F_{A, \mathrm{SM}}^{(s)}=(-7.9-0.1 i) \times 10^{-9}$.

In the MSSM, the dominant contributions to $B_{q} \rightarrow \mu^{+} \mu^{-}$are the $\tan ^{3} \beta$ enhanced wave function correction diagrams with a heavy higgs propagator, see figure 6 . Here gluinos, charginos and neutralinos can run in the loop, with both CP even and odd higgs states along the penguin line. For simplicity, we will quote the result only for gluinos; the answer for the others is very similar. As our higgs states are heavy, we use the relation $m_{A}^{2} \approx m_{H}^{2}$ to simplify expressions.

These diagrams contribute to $C_{S}^{M N}$, and the discussion is similar to that of $b \rightarrow q \gamma$. $C_{S}^{L N}$ transforms in the $(\mathbf{3}, \overline{\mathbf{3}})$ of $\mathrm{SU}(3)_{D} \times \mathrm{SU}(3)_{Q}$ while $C_{S}^{R N}$ transforms in the $(\mathbf{3}, \overline{\mathbf{3}})$ of $\mathrm{SU}(3)_{Q} \times \mathrm{SU}(3)_{D}$. Thus as before, the latter is zero thanks to $\chi \mathrm{FV}$ and we can focus on the former. Here we can afford to work at $v=0$ since we are not concerned with $Z$-penguins. 


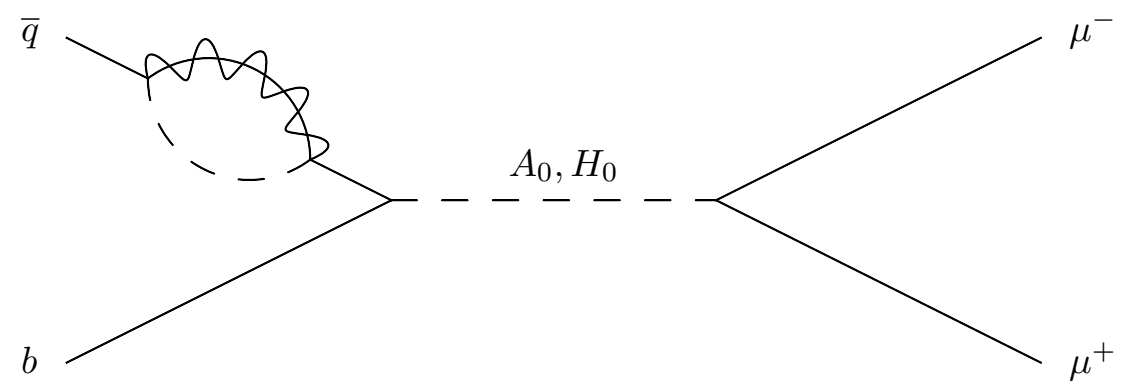

Figure 6. Wave function correction diagrams that provide the leading, $\tan ^{3} \beta$ contributions to $B_{q} \rightarrow \mu^{+} \mu^{-}$. Loop can be a gluino, chargino, or neutralino.

With only left-handed squarks propagating in the loops, by symmetries the answer must be of the form:

$$
C_{S, 3 a}^{L M}=\frac{\left(K y_{d}\right)_{3 a}}{\kappa^{2} m_{S}^{2}} f_{5}=\hat{\kappa}_{3} \hat{\kappa}_{a} \frac{y_{b}}{m_{S}^{2}} f_{5}
$$

where $a=2$ for $B_{s} \rightarrow \mu \mu$ and $a=1$ for $B_{d} \rightarrow \mu \mu$. Now the Yukawa coupling needed on symmetry grounds arises from the Higgs-quark-quark coupling. Explicit computation gives

$$
C_{S, \tilde{g}}^{L R}=\hat{\kappa}_{3} \hat{\kappa}_{a} \frac{m_{b} \tan \beta}{m_{W}} \frac{m_{\mu} \tan ^{2} \beta}{m_{W}} \frac{4 \alpha_{2} \alpha_{s}}{3 m_{A}^{2}} \frac{\mu}{m_{S}} f_{\tilde{g}}^{B_{q} \rightarrow \mu^{+} \mu^{-}, \mathrm{h}-\mathrm{peng}}\left(x_{q}, x_{g}\right)
$$

where $x_{g}=m_{\tilde{g}}^{2} / m_{0}^{2}$ and $x_{q}=m_{S}^{2} / m_{0}^{2}$ and the loop function (see appendix E) is $f_{\tilde{g}}^{B_{q} \rightarrow \mu^{+} \mu^{-} \text {,h-peng }}\left(x_{q}, x_{g}\right) \sim 0.1$. Since $m_{A} \sim m_{\mathrm{SUSY}}$, this leading contribution is of the form (3.27) as expected.

From the above expressions, we can translate to the phenomenologically useful parameters

$$
\begin{aligned}
& F_{P}^{(s)}=\frac{m_{B_{s}}^{3}}{m_{b}+m_{s}} C_{S}^{L R} \sim\left(2 \times 10^{-10}\right) \hat{\kappa}_{3} \hat{\kappa}_{2} \\
& F_{P}^{(d)}=\frac{m_{B_{d}}^{3}}{m_{b}+m_{d}} C_{S}^{L R} \sim\left(2 \times 10^{-10}\right) \hat{\kappa}_{3} \hat{\kappa}_{1} .
\end{aligned}
$$

These translate into maximum deviations from the SM branching fractions by

$$
\begin{aligned}
& {\left[\Delta \operatorname{BR}\left(B_{s} \rightarrow \mu^{+} \mu^{-}\right)\right]_{\mathrm{MAX}} \simeq X_{s}\left\{2 \operatorname{Re}\left[F_{A, \mathrm{SM}}^{(s) *} F_{P}^{(s)}\right]\right\} \sim 9 \times 10^{-11}} \\
& {\left[\Delta \operatorname{BR}\left(B_{d} \rightarrow \mu^{+} \mu^{-}\right)\right]_{\mathrm{MAX}} \simeq X_{d}\left\{2 \operatorname{Re}\left[F_{A, \mathrm{SM}}^{(d) *} F_{A}^{(d)}\right]\right\} \sim 1.2 \times 10^{-11}}
\end{aligned}
$$

These deviations are an order of magnitude smaller than the uncertainties on their respective measurements, and therefore neither $B_{s} \rightarrow \mu^{+} \mu^{-}$nor $B_{d} \rightarrow \mu^{+} \mu^{-}$place any meaningful constraints on our models. ${ }^{9}$

\footnotetext{
${ }^{9}$ In order for $B_{s} \rightarrow \mu^{+} \mu^{-}$to place any meaningful constraint, one would have to take tan $\beta$ much larger than the $\tan \beta \sim 10$ that we have assumed in this paper, e.g., $\tan \beta \gtrsim 30$.
} 


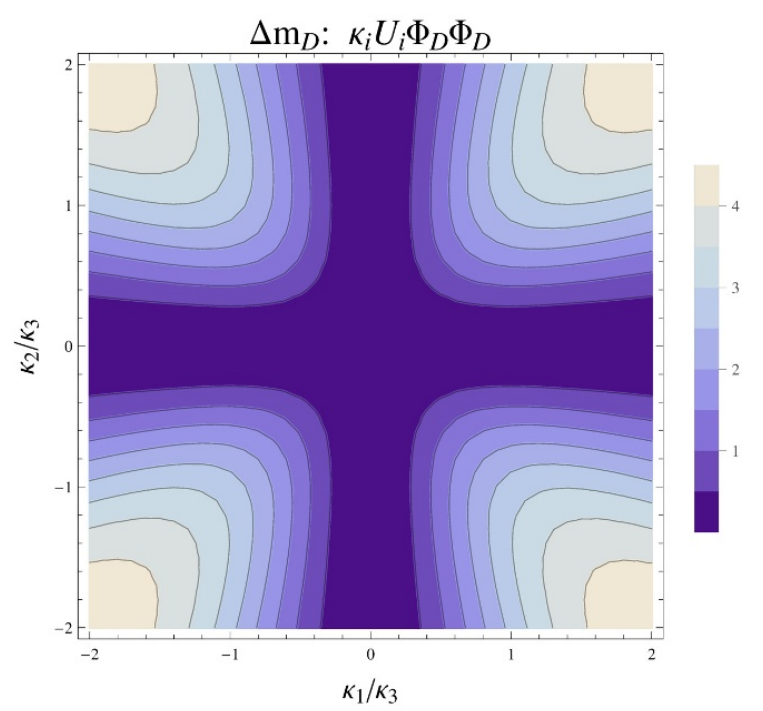

Figure 7. Plot of $\Delta m_{D}$ meson mixing observable for a $U$-class model.

\section{$3.2 \quad$ U-class models}

As discussed in section 2.1, all $U$-class $\chi \mathrm{FV}$ models receive very few constraints from flavor observables. First, according to the $\chi \mathrm{FV}$ texture of $U$-class models (2.5), all flavorviolation is restricted to the up-squark sector only. As most potentially constraining flavor observables have external down-type quarks, chargino diagrams are required for sensitivity to flavor-violation. However, as is further shown in (2.5), there is no flavor-violation in the $L L$ block; thus pure wino diagrams cannot contribute. Higgsino diagrams introduce Yukawa couplings which suppress contributions to down sector observables enough that none of these would be remotely constraining in the foreseeable future. We have verified all of these general results in the context of our type I EGMSB models.

There can be constraints from the $D$-meson system. Contributions to $\Delta m_{D}$ are as in the $Q$-class models, only now with the $C_{V}^{R R}$ contribution dominating, so we expect very similar constraints from $\Delta m_{D}$. This is indeed shown in figure 7. Again, although the contour of 2 does not represent a $95 \%$ confidence level exclusion, values larger than 1 necessitate a cancellation between the standard model and the new physics contributions. It should be noted that a viable possibility is that the standard model contribution is in fact much smaller than the observed value, and the contour of one is actually where EGMSB entirely accounts for the $\Delta m_{D}$ measurement. As with the $Q$-class models, the sensitivity vanishes as either $\kappa_{1} \rightarrow 0$ or $\kappa_{2} \rightarrow 0$, and increases most rapidly along the diagonals.

\section{Conclusions}

\subsection{Summary and discussion}

In this work, we performed a detailed investigation into the precision flavor constraints on extended GMSB models. These models, where the usual GMSB contributions are augmented by direct matter-messenger couplings, are well motivated in light of the recent 
discovery of a Higgs boson near $125 \mathrm{GeV}$. However, since these models are not necessarily $\mathrm{MFV}$, in their full, three-family generalizations they could be potentially dangerous from the point of view of flavor.

Our work required a computer program that could turn general MSSM spectra into precision flavor observables. We found that existing programs had various limitations - they either assumed MFV, were numerically unstable, or had incorrectly transcribed formulas from the literature. This motivated us to develop FormFlavor, a comprehensive package that computes flavor observables ab initio starting from the Feynman rules, and uses a modular framework that enables us to add new observables in a uniform and straightforward way.

Using FormFlavor, we studied the flavor constraints on the three-family generalizations of the EGMSB models of [20]. The results we encountered from this systematic study were interesting and unexpected. We found that despite the introduction of $\mathcal{O}(1)$ flavorviolating couplings, there are currently very few constraints on EGMSB models. To validate the numerical results of FormFlavor, we compared them in detail with analytic formulas for the Wilson coefficients derived using a combination of flavor symmetry arguments and direct calculation, and we found excellent agreement.

The mild flavor constraints in these models are illustrated in the summary plot of figure 8. $U$-class models only receive constraints from $\Delta m_{D}$. $Q$-class models are constrained by $\Delta m_{K}$ and $\Delta m_{D}$ in the corners of the plot, while the radiative $b \rightarrow s \gamma$ and $b \rightarrow d \gamma$ each exclude a single bubble near $\kappa_{2}=-\kappa_{3}$ and $\kappa_{1}=-\kappa_{3}$, respectively. We note that the excluded region from $b \rightarrow s \gamma$ is largely due to the current $\sim 1 \sigma$ discrepancy between the theoretical prediction and the measurement, which new theoretical work suggests will disappear [54].

The results from these models may seem at odds with the SUSY flavor problem. We have argued that the mildness of the flavor violation in these models originates from the fact that they obey the "chiral flavor violation" ansatz, whereby flavor is violated only by the Yukawas and spurions of a single SU(3) of the full SU(3) ${ }^{5}$ SM flavor symmetry. We showed that $\chi \mathrm{FV}$ prevents many of the most problematic contributions to flavor observables from arising in the MSSM, and allows for $\mathcal{O}(1)$ flavor-violation in EGMSB models.

\subsection{Future constraints}

Although constraints are currently very mild, there is immense potential to further probe these models in the near future.

- For $\Delta m_{K}$, short-distance predictions exist with moderate uncertainty, i.e., (3.1 \pm $1.2) \times 10^{-15} \mathrm{GeV}$ [58], but the long-distance contributions are currently unknown. However, an accurate, full calculation on the lattice, including both long- and shortdistance contributions, may be coming in the near future, as promising preliminary work on the subject shows $[59,60]$.

- Expected incremental improvements to the theoretical uncertainty of the standard model prediction could make $\Delta B_{d}$ into a constraining observable soon. Estimates 

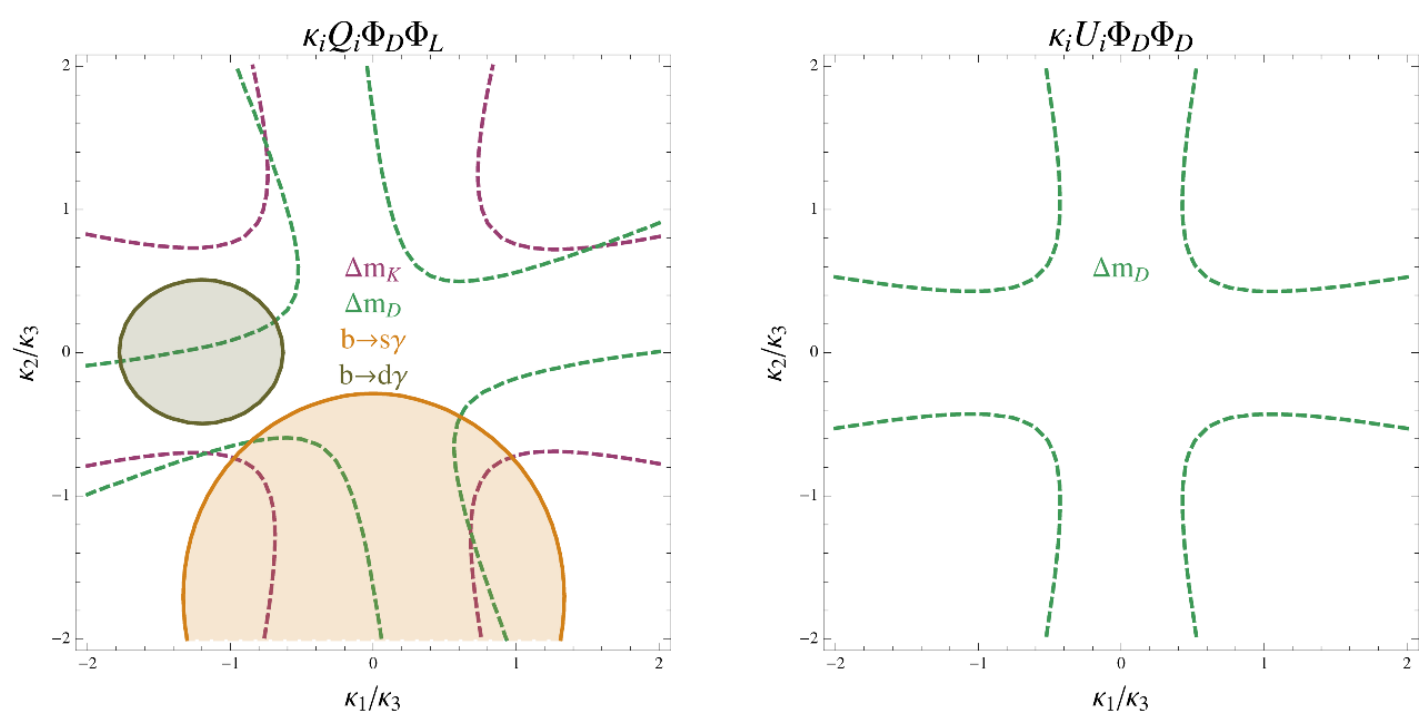

Figure 8. Flavor sensitivity for a $Q$-class (left) and $U$-class (right) model. Shaded regions of this EGMSB parameter space are excluded to $95 \%$ confidence level by current measurements of flavor observables as described in the text. Dashed lines indicate the locations where the EGMSB contribution to $\Delta m_{K}$ and $\Delta m_{D}$ are equal to the measured value (as these currently cannot provide a genuine exclusion due to poorly controlled theoretical uncertainties). In both cases, observables not shown are not constraining.

suggest that both the bag parameter, $B_{V, B_{d}}^{L L}$, and the relevant CKM elements could be calculated on the lattice to significantly improved levels by 2018 [61].

- Belle II [62] is expected to make significantly improved measurements to both $b \rightarrow s \gamma$ and $b \rightarrow d \gamma$ [63], which would allow for both of these observables to constrain more of the parameter space.

- NA62 [64] at CERN is projected to be able to measure $\operatorname{BR}\left(K^{ \pm} \rightarrow \pi^{ \pm} \nu \bar{\nu}\right)$ to a precision of about $10 \%$ [65] of the SM value. This is an improvement of more than an order of magnitude relative to the current measurement. In a few years, $K^{+} \rightarrow \pi^{+} \nu \bar{\nu}$ will be one of the the most sensitive flavor observable to $Q$-class models. Perhaps more importantly, if NA62 were to measure a deviation from the standard model prediction, this model would provide a significant motivation to invest in an experiment like ORKA [66], that would be able to hone in on the parameter space.

Due to these potentially significant theoretical and experimental improvements, much of the parameter space in $Q$-class models could be probed in just a few years. The projected sensitivities are shown in figure 9. Although $Q$-class models have an exciting future in flavor, $U$-class models remain completely unconstrained. Short of lattice predictions for $\Delta m_{D}$, no observables in the current program are sensitive to these these models. Charm factories and precision top studies could someday explore this space in, for instance, $c \rightarrow u \gamma$ or $t \rightarrow c / u \gamma$. However, for the moment, $U$-class models are resilient against flavor constraints. 


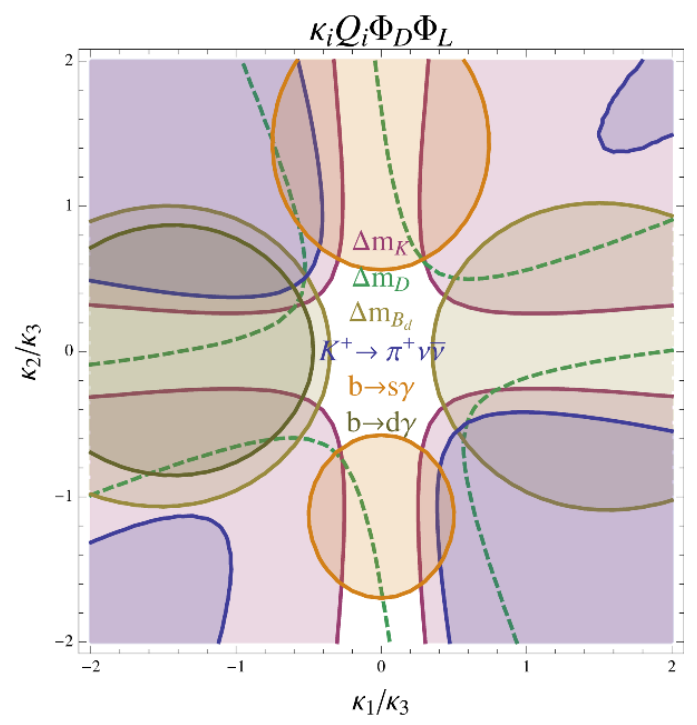

\begin{tabular}{|c|c|}
\hline Observable & Projected Accuracy \\
\hline$\Delta m_{K}$ & $10 \%_{\text {th. }}[67]$ \\
\hline$\Delta m_{B_{d}}$ & $10 \%_{\text {th. }}[61]$ \\
\hline$\Delta m_{B_{s}}$ & $5 \%_{\text {th. }}[61]$ \\
\hline$\Delta m_{D}$ & None \\
\hline$B r\left(K^{+} \rightarrow \pi^{+} \nu \bar{\nu}\right)$ & $10 \%_{\text {exp. }}[61]$ \\
\hline$B r\left(B \rightarrow X_{s} \gamma\right)$ & $7 \%_{\text {exp. }}[63]$ \\
\hline$B r\left(B \rightarrow X_{d} \gamma\right)$ & $24 \%_{\text {exp. }}[63]$ \\
\hline $\operatorname{Br}\left(B_{s} \rightarrow \mu^{+} \mu^{-}\right)$ & $15 \%_{\text {exp. }}[61]$ \\
\hline $\operatorname{Br}\left(B_{d} \rightarrow \mu^{+} \mu^{-}\right)$ & $35 \%_{\text {exp. }}[61]$ \\
\hline
\end{tabular}

Figure 9. Future projections for flavor constraints on EGMSB in the $\sim 3-5$ year range. In the figure, the errors have been updated to the fractional values in the accompanying table, and the central values have set equal between theory and experiment. Depicted is the same $Q$-class model from figure 8 . The dashed green line, which is unchanged from figure 8, indicates where the EGMSB contribution to $\Delta m_{D}$ is equal to the measured value. Observables not shown are not constraining.

\subsection{Future directions}

There are many avenues for future investigations. Here we list a few.

- As we alluded to earlier, the $\chi \mathrm{FV}$ ansatz is a general paradigm, and it provides a novel and realistic solution to the SUSY flavor problem. This texture, its possible origins, and consequences are worthy of further study [51].

- Another interesting question is that of $\mathrm{CP}$ violation in these models. The goal of this work was to focus on the SUSY flavor problem, and so the EGMSB couplings were intentionally assumed to be real. Allowing for $\arg \kappa_{i} \neq 0$ would give nontrivial contributions to CP-violating observables, some of which (such as $\epsilon_{K}$ and the neutron EDM) are typically extremely constraining. An interesting question is to what extent the $\chi \mathrm{FV}$ texture protects EGMSB models from the SUSY CP problem before there is any conflict with data. We plan to study this in detail in an upcoming work [35].

- The possibility of heavily mixed squarks allows for very interesting collider signatures [68-71]. These EGMSB models provide a flavor-safe proof-of-concept motivation for experimental searches at ATLAS and CMS.

- While we made some effort to ensure that the flavor-violating $A$-terms do not destabilize the vacuum (see the discussion in appendix A), it would be interesting to study in more detail the vacuum stability of these flavor-violating EGMSB models, along the lines of [72]. 


\section{Acknowledgments}

We are especially grateful to W. Altmannshofer for valuable discussions and comments on the draft. We also thank A. El-Khadra, S. Gori, S. Martin, G. Perez, K. Pitts, and F. Staub for useful discussions. The work of J.A.E. was supported in part by the National Science Foundation under Grant No. PHYS-1066293 and the hospitality of the Aspen Center for Physics. The work of D.S. was supported in part by a DOE Early Career Award and a Sloan Foundation Fellowship. The work of A.T. was supported in part by DOE grant DOE-SC0010008.

\section{A Details of the deformation and parameter scan}

In this appendix, we detail our parameter scan. In particular, we specify the deformation about the best points in [20] that we use. Our type I models are characterized by the following parameter space:

$$
\left(\kappa_{1}, \kappa_{2}, \kappa_{3}, \Lambda / M, \Lambda\right)
$$

In [20], the models were studied at the $\kappa_{1}=\kappa_{2}=0$ point. For each $\kappa_{3}$ and $\Lambda / M, \Lambda$ (which sets the overall scale of the superpartner spectrum) was increased until $m_{h}=125 \mathrm{GeV}$ was achieved. Then the fine-tuning of the point was estimated and the region of least fine-tuning in $\left(\kappa_{3}, \Lambda / M\right)$ space was identified. Our aim is to investigate these regions in the presence of nontrivial flavor violation, parametrized by $\kappa_{1}$ and $\kappa_{2}$. A full optimization of the fine-tuning would involve a five-dimensional scan in the $\left(\kappa_{1}, \kappa_{2}, \kappa_{3}, \Lambda / M, M\right)$ parameter space. Such an endeavor is not computationally feasible and moreover is unnecessary. The main question we intend to explore is how qualitatively dangerous the flavor-physics contributions are in these EGMSB models. In principle, this question can be answered using a simpler scan where we essentially fix $\left(\kappa_{3}, \Lambda / M, M\right)$ as in [20] and then vary $\kappa_{1}, \kappa_{2}$. However, there are a number of subtleties to take into account when doing so, most of which stem from the fact that the points from [20] tended to have light stops as a result of a cancellation (this is unsurprising due to the tuning involved).

- We must be careful to choose $\left(\kappa_{3}, \Lambda / M\right)$ such that the point at $\kappa_{1}=\kappa_{2}=0$ is unconstrained by Run I searches. In practice, to get a model that is not constrained by the LHC and has a good tuning value, we will lower $\frac{\Lambda}{M}$ away from the least-tuned point identified in [20]. This increases the fine-tuning required in the models only by about $10 \%$. The parameters we choose (at the origin) are $\kappa_{3}=0.858, N=6$, $\frac{\Lambda}{M}=0.347$, and $M=312 \mathrm{TeV}$ for I.9, and $\kappa_{3}=0.908, N=3, \frac{\Lambda}{M}=0.290$, and $M=350 \mathrm{TeV}$ for I.13. The spectra are shown in figure 1 .

- Despite our focus on CP conserving observables in this work, there could, in principle, be significant constraints from $\epsilon_{K}$ introduced solely through the CP violating phase of the CKM. ${ }^{10}$ In order to avoid this constraint, in our $Q$-class models, we

\footnotetext{
${ }^{10}$ We thank W. Altmannshofer for bringing this to our attention.
} 
choose $\kappa_{1}, \kappa_{2}$ and $\kappa_{3}$ to align with the low-energy down, strange and bottom quark, respectively. Thus in our choice of interaction basis, the down Yukawa is diagonal, but the up Yukawa is multiplied by $V_{C K M}$. We ignore the small differences with our previous work where the alignment was with the top quark. The $U$-class models retain alignment with the up-type quarks.

- Turning on $\kappa_{1}$ and $\kappa_{2}$ while holding fixed the other parameters can significantly modify the spectrum, as shown in (2.6). At the least finely-tuned points, where a cancellation results in a stop lighter than the other squarks, this can either lead to stop tachyons, or it can lead to the stops being so heavy that the hypercharge tadpole contribution to the RG running quickly drives the sleptons tachyonic. To avoid these undesirable features, as we turn on $\kappa_{1,2}$, we fix $\Lambda$, but vary $M$, so that the the oneloop contribution adapts in such a way that the lightest squark eigenvalue of the LL block in $Q$-class or RR block in $U$-class models is held fixed. This deformation enables us to prevent the interesting flavor-violation from vanishing and to maintain the squark masses at sensible values.

- Additionally, as can be seen from (2.6), if we were to fix $\kappa_{3}$ and turn on $\kappa_{1,2}$, then the "net" $A$-term would increase. The "net" $A$-term in the type I models is aligned with the lightest eigenvalue direction of the rank 1 block, i.e., in $Q$-class models,

$$
\mathcal{L} \supset-\frac{\kappa \kappa_{3} y_{t} \Lambda}{16 \pi^{2}} H_{u} \tilde{Q}_{S} \tilde{t}_{R} .
$$

The $A$-terms in all orthogonal squark directions vanish in the third-generation dominant limit. Increasing $A / m_{S}$ is potentially dangerous for vacuum stability [72]. In order to avoid these issues, we require that this "net" $A$-term remains constant. To achieve this we fix $\kappa_{3} \kappa$ everywhere in the parameter space so that as we increase $\kappa_{1}$ and $\kappa_{2}, \kappa_{3}$ decreases to compensate.

- Finally, this deformation of $\kappa_{1,2,3}$ will also affect the Higgs mass. The one-loop corrections to the Higgs mass in the presence of general flavor violation have been computed in $[73,74]$, and our preliminary studies of these suggest that $m_{h}$ may drift down by several $\mathrm{GeV}$ as we move out in $\kappa_{1,2}$. However, the two-loop corrections are not yet known, and if the usual non-flavor-violating MSSM is any guide, these are likely to be important for an accurate determination of the Higgs mass. While it would be interesting to study this further, it is beyond the scope of the work. At the very least, one could imagine increasing the overall scale of the superpartners in order to compensate for any decrease in the Higgs mass. This would only serve to further alleviate the flavor constraints, so the qualitative value of $\chi \mathrm{FV}$ to these EGMSB models is unaffected.

Across a grid in $\left(\kappa_{1}, \kappa_{2}\right)$ constructed via this deformation of the benchmark point, we generate the soft spectrum at the messenger scale. All of the couplings and soft masses are then evolved down from the messenger scale to the SUSY scale using the fully general 


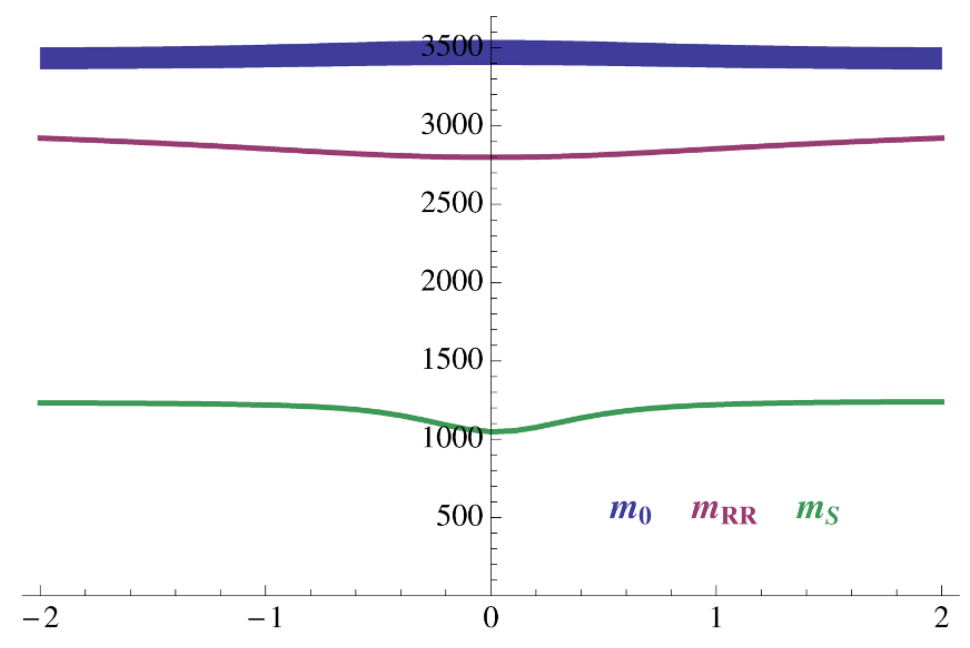

Figure 10. The spectra of squark masses (in GeV) for model I.9 as we move in $\kappa_{1}=\kappa_{2}$ Aside from the three lightest eigenvalues, $m_{S, \text { up }}=m_{S, \text { down }}$, and $m_{R R}$, the other nine squark eigenvalues all fall in the range of the blue band near $3.5 \mathrm{TeV}$. The width of the band is largely due to the $\mathrm{SU}(2)$ GMSB contributions to the left-handed squarks.

$3 \times 3$ MSSM $\beta$-functions. ${ }^{11}$ At the SUSY scale, we apply the BMPZ QCD threshold corrections to the squarks and gluinos [75]. Finally, we use our new Mathematica package FormFlavor to compute the Wilson coefficients, RG evolve these coefficients down to the scale of interest (e.g., $m_{b}$ ) for each flavor observable, and compute the contributions to each flavor observable there. (The details of FormFlavor are briefly explained in appendix B and will be further fleshed out when the package is made publicly available [39].)

\section{B Details of FormFlavor}

FormFlavor is a general, modular flavor package written in Mathematica that takes general MSSM spectra as inputs and calculates a variety of flavor observables in situ starting from the Feynman rules. The package is flexible - new flavor observables can be straightforwardly added to it and, although currently implemented only for the MSSM, it can be extended to other models readily. Also, since processes are calculated in a uniform manner from first principles (rather than hardcoding formulas from the literature), the reliability of the code is greatly enhanced.

In FormFlavor, the process under consideration is specified in FeynArts [36] and all the relevant topologies for the various sparticle mediators are generated there. These topologies are then converted into their respective amplitudes via FormCalc [37]. The FormCalc output is analytically transformed into a particular Wilson operator basis for each process of interest, and the Wilson coefficients are extracted. Having performed this once for an observable the analytic expressions may be written to a process file and used in subsequent runs. The Wilson coefficients may then be piped into functions that calculate the flavor observables.

\footnotetext{
${ }^{11}$ For simplicity, we do not run back and forth between the IR and UV to better specify the scale.
} 
The main components of FormFlavor are as follows:

- Automated generation of one-loop diagrams for flavor processes (using FeynArts)

- Automated calculation of Wilson coefficients from Feynman diagrams (using FormCalc)

- Library of analytic one-loop integral functions

- Routines for general MSSM spectrum input (SLHA2 [76] compatible)

- Routines for converting Wilson coefficients to flavor observables. (This step contains hardcoded formulas from the literature and is not MSSM specific.) The following processes are currently implemented:

CP-conserving observables

* Meson Mixings [52, 77]: $\Delta M_{K}$ [78], $\Delta M_{D}$ [79], $\Delta M_{B_{d}}$ and $\Delta M_{B_{s}}[40,80]$

* Leptonic decays: $K^{+} \rightarrow \pi^{+} \nu \bar{\nu}[41,81,82], B_{s} \rightarrow \mu^{+} \mu^{-}$and $B_{d} \rightarrow \mu^{+} \mu^{-}\left[46,55^{-}\right.$ $57]$

* Radiative Decays: $b \rightarrow s \gamma[42,83], b \rightarrow d \gamma[44,84]$

CP-violating observables

* Meson Mixings: $\epsilon_{K}$

* Leptonic decays: $K^{0} \rightarrow \pi^{0} \nu \bar{\nu}$

* Electric dipole moments: neutron-EDM

- MSSM RGE - Full three-family MSSM renormalization group evolution, including arbitrary CP-phases, implemented with the aid of SARAH [85]

This powerful flavor package will be released to the public and described in more detail in an upcoming work [39].

\section{Soft terms for type I squark models}

In this appendix, we will specialize the fully general EGMSB formulas from [20] to the case of the type I squark models with general flavor-violating couplings $\kappa_{i}$. These were quoted in (2.6) for the $Q$-class models,

$$
W_{\mathrm{EGMSB}}=\kappa_{i} Q_{i} \sum_{A=1}^{N} \Phi_{A} \tilde{\Phi}_{A}
$$

and we again focus on this case here. To translate to $U$-class models, one must take $U \leftrightarrow Q$, $y_{u} \rightarrow y_{u}^{\dagger}, A_{\tilde{u}} \rightarrow A_{\tilde{u}}^{\dagger}$ and $K \rightarrow K^{T}$ in all expressions. 
Before we begin, we must address a convention difference between this paper and that of [20] concerning the treatment of the right-handed quark chiral superfields $\bar{U}, \bar{D}$. In this paper, the Yukawas, squark mass matrices and $A$-terms are defined as:

$$
\begin{aligned}
& W \supset H_{u} \bar{U}_{i} y_{u, i j} Q_{j}+H_{d} \bar{D}_{i} y_{d, i j} Q_{j} \\
& \mathcal{L} \supset \tilde{Q}_{i}^{*} \delta m_{Q, i j}^{2} \tilde{Q}_{j}+\tilde{U}_{i}^{*} \delta m_{U, i j}^{2} \tilde{U}_{j}+\tilde{D}_{i}^{*} \delta m_{D, i j}^{2} \tilde{D}_{j} \\
& \quad+\left(H_{u} \tilde{U}_{i}^{*} A_{\tilde{u}, i j} \tilde{Q}_{j}+H_{d} \tilde{D}_{i}^{*} A_{\tilde{d}, i j} \tilde{Q}_{j}+\text { h.c. }\right)
\end{aligned}
$$

Thus, the Yukawas, $A$-terms, $m_{U}^{2}$ and $m_{D}^{2}$ are all transposed relative to those of [20]. Note that in these conventions, $\tilde{U}^{*}, \tilde{D}^{*}$ are the scalar components of the chiral supermultipliets $\bar{U}, \bar{D}$.

The starting point of our derivation of (2.6) is eq. (2.19) in [20], which we repeat here for convenience:

$$
\begin{aligned}
A_{a b}= & -\frac{1}{32 \pi^{2}} d_{a}^{B C} \lambda_{a B C}^{*} \lambda_{b B C} \Lambda \\
\delta m_{a b}^{2}= & \frac{1}{256 \pi^{4}}\left(d_{a}^{B C} d_{B}^{c D} \lambda_{a B C}^{*} \lambda_{b C E} \lambda_{c B D} \lambda_{c D E}^{*}+\frac{1}{4} d_{a}^{B C} d_{b}^{D E} \lambda_{a B C}^{*} \lambda_{c B C} \lambda_{c D E}^{*} \lambda_{b D E}\right. \\
& \left.\quad-\frac{1}{2} d_{a}^{c d} d_{c}^{B C} y_{a c d}^{*} y_{b d e} \lambda_{c B C} \lambda_{e B C}^{*}-d_{a}^{B C} C_{r}^{a B C} g_{r}^{2} \lambda_{a B C}^{*} \lambda_{b B C}\right) \Lambda^{2}
\end{aligned}
$$

Here $a, b, \ldots$ run over all MSSM fields (including all gauge and flavor degrees of freedom). Meanwhile $A, B, \ldots$ run over all messenger fields similarly. In this appendix, we will use $i, j, \ldots$ to denote MSSM flavor indices.

From (C.3), we immediately obtain the bilinear $A$-terms after summing over the $N$ messenger multiplets and substituting $\lambda_{a B C} \rightarrow \kappa_{i}$ and $d_{a}^{B C} \rightarrow d_{Q} \propto N$

$$
A_{Q, i j}=-\frac{1}{16 \pi^{2}} d_{Q} \kappa_{i}^{*} \kappa_{j} \Lambda
$$

This becomes the trilinear $A$-term $A_{\tilde{u}}$ used in the paper via:

$$
A_{Q, i j} F_{Q_{i}}^{\dagger} \tilde{Q}_{j} \rightarrow y_{u, i k} A_{Q, k j} H_{u} \tilde{U}_{i}^{*} \tilde{Q}_{j} \equiv A_{\tilde{u}, i j} H_{u} \tilde{U}_{i}^{*} \tilde{Q}_{j}
$$

Next let's consider the EGMSB contributions to the soft mass-squareds, starting with $m_{Q}^{2}$. From (C.3), we obtain:

$$
\begin{aligned}
\delta m_{Q, i j}^{2} & =\frac{1}{256 \pi^{4}}\left(d_{Q} d_{\phi} \kappa_{i}^{*} \kappa_{j} \kappa_{k} \kappa_{k}^{*}+d_{Q}^{2} \kappa_{i}^{*} \kappa_{k}^{*} \kappa_{k} \kappa_{j}-2 d_{Q} C_{r} g_{r}^{2} \kappa_{i}^{*} \kappa_{j}\right) \Lambda^{2} \\
& =\frac{d_{Q}}{256 \pi^{4}}\left(\left(d_{\phi}+d_{Q}\right) \kappa^{2}-2 C_{r} g_{r}^{2}\right) K_{i j} \Lambda^{2}
\end{aligned}
$$

In the first line, we have introduced $d_{B}^{c D} \rightarrow \frac{1}{2} d_{\phi}$, and we have used the fact that $Q$ is the only MSSM field coupling to the messengers to set $d_{a}^{c d} d_{c}^{B C}$ to zero. Additionally, as $Q$ couples directly to the messengers, there is a one-loop term suppressed by $\frac{\Lambda^{2}}{M^{2}}[13]$,

$$
\delta\left(m_{Q, i j}^{1-\text { loop }}\right)^{2}=-\frac{16 \pi^{2}}{3} h\left(\frac{\Lambda}{M}\right) \frac{\Lambda^{2}}{M^{2}} \frac{d_{Q} K_{i j} \Lambda^{2}}{256 \pi^{4}}
$$


where $h(x)$ is a loop function given by

$$
h(x)=\frac{3}{x^{4}}((x-2) \ln (1-x)-(x+2) \ln (1+x))=1+\frac{4}{5} x^{2}+\mathcal{O}\left(x^{4}\right) .
$$

The EGMSB contribution to $m_{H_{u}}^{2}$ is much simpler. Here only the third term of $\delta m_{a b}^{2}$ in (C.3) contributes:

$$
\delta m_{H_{u}}^{2}=-\frac{3 d_{Q}}{256 \pi^{4}} y_{u, i j}^{*} y_{u, i k} \kappa_{j} \kappa_{k}^{*} \Lambda^{2}=-\frac{3 d_{Q}}{256 \pi^{4}} \operatorname{Tr}\left[y_{u} K y_{u}^{\dagger}\right] \Lambda^{2}
$$

where we have used $d_{H}^{Q U}=3$.

Lastly, the EGMSB contribution to $m_{U}^{2}$ also comes from just the third term of $\delta m_{a b}^{2}$ :

$$
\delta m_{U, i j}^{2}=-\frac{d_{U}^{Q H} d_{Q}}{256 \pi^{4}} y_{u, i k}^{*} y_{j \ell} \kappa_{k} \kappa_{\ell}^{*} \Lambda^{2}=-\frac{d_{U}^{Q H} d_{Q}}{256 \pi^{4}}\left(y_{u} K y_{u}^{\dagger}\right)_{j i} \Lambda^{2}
$$

where $d_{U}^{Q H}=2$. Taking into account the need to transpose $m_{U}^{2}$ to translate between the conventions of [20] and those of this paper, we obtain the correct result quoted in (2.6).

\section{Formulas for flavor observables}

In this appendix, we collect formulas from the literature for the various flavor observables considered in this work. Along the way, we will streamline the different notations scattered throughout the literature into a uniform convention.

The uniform operator basis we will use was introduced in the text; we repeat it here for convenience. For dimension 5, we have:

$$
\begin{aligned}
& \mathcal{O}_{A}^{M}\left(f_{1}, f_{2}\right)=e \bar{f}_{1} \sigma^{\mu \nu} P_{M} f_{2} F_{\mu \nu} \\
& \mathcal{O}_{G}^{M}\left(f_{1}, f_{2}\right)=g \bar{f}_{1} \sigma^{\mu \nu} P_{M} f_{2} G_{\mu \nu}
\end{aligned}
$$

For dimension 6, we have:

$$
\begin{aligned}
& \mathcal{O}_{S}^{M N}\left(f_{1}, f_{2}, f_{3}, f_{4}\right)=\left(\bar{f}_{1} P_{M} f_{2}\right)\left(\bar{f}_{3} P_{N} f_{4}\right) \\
& \mathcal{O}_{V}^{M N}\left(f_{1}, f_{2}, f_{3}, f_{4}\right)=\left(\bar{f}_{1} \gamma^{\mu} P_{M} f_{2}\right)\left(\bar{f}_{3} \gamma_{\mu} P_{N} f_{4}\right) \\
& \mathcal{O}_{T}^{M N}\left(f_{1}, f_{2}, f_{3}, f_{4}\right)=\left(\bar{f}_{1} \sigma^{\mu \nu} P_{M} f_{2}\right)\left(\bar{f}_{3} \sigma_{\mu \nu} P_{N} f_{4}\right)
\end{aligned}
$$

where $M, N=L, R . P_{R}=\frac{1}{2}\left(1+\gamma_{5}\right), P_{L}=\frac{1}{2}\left(1-\gamma_{5}\right)$ are projection operators, $\sigma^{\mu \nu}=$ $\frac{1}{2}\left[\gamma^{\mu}, \gamma^{\nu}\right]$ and if $f_{i}$ carry color indices, they are contracted within a bilinear factor.

The general effective Hamiltonian is then:

$$
H_{\mathrm{eff}}\left(f_{i}\right)=\sum C_{X}^{M}\left(f_{i}\right) \mathcal{O}_{X}^{M}\left(f_{i}\right)+\sum C_{X}^{M N}\left(f_{i}\right) \mathcal{O}_{X}^{M N}\left(f_{i}\right)
$$

where the sums runs over a complete basis of independent operators. 


\begin{tabular}{|c|ccc|ccccc|c|}
\hline Meson & $m_{X}(\mathrm{GeV})$ & $f_{X}(\mathrm{GeV})$ & $R_{X}$ & $B_{V}^{L L}$ & $B_{V}^{L R}$ & $B_{S}^{L R}$ & $B_{S}^{L L}$ & $B_{T}^{L L}$ & $\left.C_{V, \mathrm{SM}}^{L L}\right|_{\mu=m_{b}}\left(\mathrm{GeV}^{-2}\right)$ \\
\hline$\Delta m_{K}$ & 0.4976 & 0.160 & 24.3 & 0.56 & 0.85 & 1.08 & 0.62 & 0.43 & - \\
$\Delta m_{D}$ & 1.8645 & 0.209 & 3.20 & 0.76 & 0.97 & 0.95 & 0.64 & 0.39 & - \\
$\Delta m_{B_{d}}$ & 5.2796 & 0.191 & 1.65 & 0.84 & 1.47 & 0.95 & 0.72 & 0.61 & $(2.34-2.20 i) \times 10^{-12}$ \\
$\Delta m_{B_{s}}$ & 5.3668 & 0.228 & 1.65 & 0.88 & 1.57 & 0.93 & 0.73 & 0.62 & $(6.96-0.26 i) \times 10^{-11}$ \\
\hline
\end{tabular}

Table 2. Properties of the meson mixing used in this work. $f_{X}$ and $B_{V, X}^{L L}$ come from the FLAG review [77]. The other four non-perturbative $B$-parameters are taken from several sources: for $\Delta m_{K}$ from [78] (at $\mu=2 \mathrm{GeV}$ ), $\Delta m_{D}$ from [79] (at $\mu=3 \mathrm{GeV}$, rescaled for a common $R_{D}$, and converted to our basis using $B_{T}^{L L}=\frac{5}{3} B_{2}-\frac{2}{3} B_{3}$ ), and for $B_{s}$ and $B_{d}$ from [80] (at $\mu=m_{b}=4.2 \mathrm{GeV}$ and converted to our basis). $\left.C_{V, S M}^{L L}\right|_{\mu=m_{b}}$ are the FormFlavor values in the CKM basis of the PDG [47].

\section{D.1 Meson Mixing $\Delta m_{X}$}

This corresponds to a $\Delta F=2$ effective Hamiltonian, with $f_{1}=f_{3}=q_{1}$, and $f_{2}=f_{4}=q_{2}$, with $\left(q_{1}, q_{2}\right)=(s, d),(c, u),(b, d),(b, s)$ for $K, D, B_{d}$ and $B_{s}$ respectively. The quantities relevant for mixing are derived from the effective Hamiltonian as

$$
\begin{aligned}
& \left\langle\bar{X}\left|H_{\mathrm{eff}}\right| X\right\rangle \equiv M_{X, 12}-\frac{i}{2} \Gamma_{X, 12} \\
& \left\langle X\left|H_{\mathrm{eff}}\right| \bar{X}\right\rangle=M_{X, 12}^{*}-\frac{i}{2} \Gamma_{X, 12}^{*}
\end{aligned}
$$

$M_{X, 12}$ and $\Gamma_{X, 12}$ are, respectively, the dispersive and absorptive parts of $\left\langle\bar{X}\left|H_{\mathrm{eff}}\right| X\right\rangle$. In terms of these quantities, the mass splitting is given by:

$$
\Delta m_{X}=2 \operatorname{Re}\left[\left(M_{X, 12}-\frac{i}{2} \Gamma_{X, 12}\right) \sqrt{\frac{M_{X, 12}^{*}-\frac{i}{2} \Gamma_{X, 12}^{*}}{M_{X, 12}-\frac{i}{2} \Gamma_{X, 12}}}\right]
$$

For $X=B_{d}$ and $B_{s}$ where $\Gamma_{X, 12} \ll M_{X, 12}$, this is well approximated by

$$
\Delta m_{X} \approx 2\left|M_{X, 12}\right| \approx 2\left|\left\langle\bar{X}\left|H_{\text {eff }}\right| X\right\rangle\right|
$$

For $X=K$ and $D$, where experimentally one finds that $M_{X, 12} / \Gamma_{X, 12}$ is approximately real, and $M_{X, 12}$ and $\Gamma_{X, 12}$ are both predicted to be approximately real in the standard CKM convention (where the CPV phase is primarily in $V_{t d}$ and $V_{u b}$ ), one has to a good approximation

$$
\Delta m_{X} \approx 2 \operatorname{Re} M_{X, 12} \approx 2 \operatorname{Re}\left\langle\bar{X}\left|H_{\text {eff }}\right| X\right\rangle
$$

Finally, the short-distance part of the matrix elements in (D.9) and (D.10) (which is all that is relevant for the $B_{q}$ systems and for new physics) are given by

$$
\begin{array}{r}
\left\langle\bar{X}\left|H_{\mathrm{eff}}\right| X\right\rangle=\frac{m_{X} f_{X}^{2}}{24}\left(8 B_{V}^{L L}\left(C_{V}^{L L}+C_{V}^{R R}\right)-R_{X}\left[4 B_{V}^{L R} C_{V}^{L R}-6 B_{S}^{L R} C_{S}^{L R}\right.\right. \\
\left.\left.+5 B_{S}^{L L}\left(C_{S}^{L L}+C_{S}^{R R}\right)+12 B_{T}^{L L}\left(C_{T}^{L L}+C_{T}^{R R}\right)\right]\right)
\end{array}
$$


where,

$$
R_{X}=\left(\frac{m_{X}}{m_{q_{1}}+m_{q_{2}}}\right)^{2}
$$

and the $B$-parameters are non-perturbative corrections that have been computed on the lattice. In the Standard Model, only $C_{V}^{L L} \neq 0$. These values and the other parameters relevant for meson mixing are shown in table 2 .

\section{$\mathrm{D} .2 \quad K^{ \pm} \rightarrow \pi^{ \pm} \nu \bar{\nu}$}

This corresponds to a dimension 6 effective Hamiltonian with $f_{1}=s, f_{2}=d, f_{3}=f_{4}=\nu_{\ell}$. The branching ratios for $K^{ \pm} \rightarrow \pi^{ \pm} \nu \bar{\nu}$ are given by [81],

$$
\operatorname{BR}\left(K^{ \pm} \rightarrow \pi^{ \pm} \nu \bar{\nu}\right)=\frac{c_{+}}{3} v^{4} \sum_{\ell=e, \mu, \tau}\left|C_{V, \ell}^{L L}+C_{V, \ell}^{R L}\right|^{2}
$$

where $v=246 \mathrm{GeV}$ and

$$
c_{+}=\frac{3 r_{K^{+}}}{2\left|V_{u s}\right|^{2}} \operatorname{BR}\left(K^{ \pm} \rightarrow \pi^{0} e^{ \pm} \nu\right)=1.35
$$

Here, the branching ratio has been included to remove dependence on the hadronic matrix element, and $r_{K^{+}}=0.901$ contains isospin violating quark mass effects and electroweak corrections computed in [86].

In the standard model, the contributions from top loops are have no sensitivity to different generations, but the charm loop contributions do. In particular, the charm contributions are the same for $e$ and $\mu$, but differ for $\tau$. Thus, the SM contribution can be expressed as,

$$
C_{V, S M, \ell}^{L L}=\frac{\alpha_{2}}{\pi v^{2}}\left(\lambda_{c} X_{c}^{\ell}+\lambda_{t} X_{t}\right)
$$

where $\lambda_{i}=V_{i s}^{*} V_{i d}, X_{t}=1.469$ [41], $X_{c}^{e}=X_{c}^{\mu}=1.055 \times 10^{-3}$, and $X_{c}^{\tau}=7.01 \times 10^{-4}$ [81].

In the absence of lepton flavor-violating new physics effects, the contributions from new physics are the same across lepton generations, i.e., $C_{V, N P, \ell}^{X Y}=C_{V, N P, \ell^{\prime}}^{X Y} \equiv C_{V, N P}^{X Y}$. We can then give a simpler form to equation (D.13)

$$
\mathrm{BR}\left(K^{ \pm} \rightarrow \pi^{ \pm} \nu \bar{\nu}\right)=c_{+} v^{4}\left|C_{V}^{L L}+C_{V}^{R L}\right|^{2}
$$

where $C_{V}^{X Y}=C_{V, N P}^{X Y}+C_{V, \mathrm{SM}}^{X Y}$, with

$$
\begin{aligned}
C_{V, \mathrm{SM}}^{R L} & =0 \\
C_{V, \mathrm{SM}}^{L L} & =\frac{\alpha_{2}}{\pi v^{2}}\left(\lambda_{c} P_{c}+\lambda_{t} X_{t}\right)=(-12.2+3.6 i) \times 10^{-11} \mathrm{GeV}^{-2} \\
P_{c} & =\left(\frac{2}{3} X_{N L}^{e}+\frac{1}{3} X_{N L}^{\tau}\right) \sim 9.37 \times 10^{-4} .
\end{aligned}
$$

Here, $P_{c}$ is the charm contribution averaged over the different neutrino flavors. Using $P_{c}$ simplifies the expression, but reduces the standard model charm contribution by about $3 \%$. This represents only a $0.3 \%$ decrease in the overall SM contribution, which is completely negligible when compared to the theoretical uncertainty. Most importantly, the interference effects with new physics are properly captured under this simplification. 


\section{D.3 $b \rightarrow s \gamma$ and $b \rightarrow d \gamma$}

These observables correspond to dimension 5 effective Hamiltonians with $f_{1}=b$ and $f_{2}=s$ or $d$. The branching ratio is given by,

$$
\operatorname{BR}(b \rightarrow q \gamma)=c_{\gamma} v^{2}\left(\left|C_{A}^{L}\right|^{2}+\left|C_{A}^{R}\right|^{2}\right)
$$

where $[87]$

$$
\begin{aligned}
c_{\gamma} & =\left(8 \pi^{2}\right)^{2} \frac{6}{\pi} \frac{\mathrm{BR}\left(b \rightarrow X_{c} e \nu\right)_{\operatorname{EXP}}}{\Phi\left|V_{c b}\right|^{2}} \frac{v^{2}}{m_{b}^{2}} \alpha_{\mathrm{EM}}=3.3 \times 10^{7} \\
\Phi & =\left|\frac{V_{u b}}{V_{c b}}\right|^{2} \frac{\mathrm{BR}\left(b \rightarrow X_{c} e \nu\right)}{\operatorname{BR}\left(b \rightarrow X_{u} e \nu\right)}=0.58
\end{aligned}
$$

where the branching ratio to charm decays is used to remove sensitivity to the hadronic matrix element, and the $\Phi$ factor is introduced to account for the nontrivial phase space factor in the compared branching ratio (due mostly to the charm quark mass).

Only $C_{A}^{L}$ contains a standard model contribution, which is,

$$
\left[C_{A, \mathrm{SM}}^{L}\right]_{b \rightarrow s \gamma}=\frac{V_{t s}^{*} V_{t b} m_{b}}{8 \pi^{2} v^{2}} X_{\mathrm{SM}} \sim(1.3+0.03 i) \times 10^{-8} \mathrm{GeV}^{-1}
$$

where $X_{\mathrm{SM}}=-0.3736$.

The observable $b \rightarrow d \gamma$ is defined completely analogously. The only difference is that the the standard model contribution differs. In particular,

$$
\left[C_{A, \mathrm{SM}}^{L}\right]_{b \rightarrow d \gamma}=\frac{V_{t d}^{*} V_{t b} m_{b}}{8 \pi^{2} v^{2}} X_{\mathrm{SM}} \sim-(2.4+1.1 i) \times 10^{-9} \mathrm{GeV}^{-1}
$$

with the same $X_{\mathrm{SM}}=-0.3736$ as above.

\section{D.4 $\quad B_{s} \rightarrow \mu^{+} \mu^{-}$and $B_{d} \rightarrow \mu^{+} \mu^{-}$}

This corresponds to a dimension 6 effective Hamiltonian with $f_{1}=b, f_{2}=s, d$ and $f_{3}=$ $f_{4}=\mu$. The branching ratio for $B_{s, d} \rightarrow \mu^{+} \mu^{-}$is $[55,56]$ :

$$
\mathrm{BR}\left(B_{i} \rightarrow \mu^{+} \mu^{-}\right)=X_{i}\left\{\left(1-\frac{4 m_{\mu}^{2}}{m_{B_{i}}^{2}}\right)\left|F_{S}^{(i)}\right|^{2}+\left|F_{P}^{(i)}+F_{A}^{(i)}\right|^{2}\right\}
$$

where

$$
\begin{aligned}
X_{i} & =\frac{f_{B_{i}}^{2}}{128 \pi m_{B_{i}} \Gamma_{B_{i}}} \sqrt{1-\frac{4 m_{\mu}^{2}}{m_{B_{i}}^{2}}} \Longrightarrow X_{s}=5.36 \times 10^{7} \text { and } X_{d}=3.97 \times 10^{7}, \\
F_{S}^{(i)} & =\frac{m_{B_{i}}^{3}}{m_{b}+m_{i}}\left(C_{S}^{L L}+C_{S}^{L R}-C_{S}^{R R}-C_{S}^{R L}\right), \\
F_{P}^{(i)} & =\frac{m_{B_{i}}^{3}}{m_{b}+m_{i}}\left(-C_{S}^{L L}+C_{S}^{L R}-C_{S}^{R R}+C_{S}^{R L}\right), \\
F_{A}^{(i)} & =2 m_{B_{i}} m_{\mu}\left(C_{V}^{L L}-C_{V}^{L R}+C_{V}^{R R}-C_{V}^{R L}\right),
\end{aligned}
$$


and $\Gamma_{B_{d}} \equiv \tau_{B_{d}}^{-1}=4.33 \times 10^{-13} \mathrm{GeV}$ and $\Gamma_{B_{s}} \equiv \tau_{B_{s}}^{-1}=4.49 \times 10^{-13} \mathrm{GeV}$. The three-loop Standard Model contribution to $B_{i} \rightarrow \mu^{+} \mu^{-}$can be expressed as [57]:

$$
F_{A, \mathrm{SM}}^{(i)}=0.4802 \times \frac{4 \alpha_{2} V_{t b}^{*} V_{t i}}{\pi} \frac{m_{\mu} m_{B_{i}}}{v^{2}} ; F_{S, \mathrm{SM}}^{(i)}=F_{P, \mathrm{SM}}^{(i)}=0
$$

so that $F_{A, \mathrm{SM}}^{(d)}=(1.5-0.6 i) \times 10^{-9}$ and $F_{A, \mathrm{SM}}^{(s)}=(-7.9-0.1 i) \times 10^{-9}$

\section{E Rank $1 \chi$ FV loop functions}

We compile some of the loop functions for our rank $1 \chi \mathrm{FV}$ approximation below.

Starting with meson-mixing, we have,

$$
\begin{aligned}
f_{\tilde{g}}^{\Delta M, \text { box }}\left(x_{g}, x_{q}\right)= & \frac{2 x_{q} \log x_{q}\left(11 x_{q}^{3}+6 x_{g} x_{q}^{2}-2 x_{g}^{2} x_{q}-13 x_{g} x_{q}-2 x_{g}^{2}\right)}{\left(x_{q}-1\right)\left(x_{q}-x_{g}\right)^{3}} \\
& +\frac{2 x_{q} x_{g} \log x_{g}\left(x_{q}-1\right)^{2}\left(2 x_{q} x_{g}+13 x_{q}-17 x_{g}^{2}+2 x_{g}\right)}{\left(x_{g}-1\right)^{3}\left(x_{q}-x_{g}\right)^{3}} \\
& -\frac{x_{q}\left(19 x_{q}^{2} x_{g}+11 x_{q}^{2}+3 x_{q} x_{g}^{2}-74 x_{q} x_{g}+11 x_{q}+8 x_{g}^{3}+3 x_{g}^{2}+19 x_{g}\right)}{\left(x_{g}-1\right)^{2}\left(x_{q}-x_{g}\right)^{2}}
\end{aligned}
$$

Here, $x_{q}=m_{S}^{2} / m_{0}^{2}$ and $x_{g}=m_{\tilde{g}}^{2} / m_{0}^{2}$; where $m_{0}$ and $m_{S}$ are the heaviest and lightest squark mass eigenvalues.

For $K^{ \pm} \rightarrow \pi^{ \pm} \nu \bar{\nu}$, there are two contributions,

$$
f_{\tilde{\chi}^{ \pm}}^{K \rightarrow \pi \nu \nu}\left(x_{\ell}, x_{2}, x_{\mu}\right)=f_{\tilde{\chi}^{ \pm}}^{K \rightarrow \pi \nu \nu, \text { box }}\left(x_{\ell}, x_{2}\right)+f_{\tilde{\chi}^{ \pm}}^{K \rightarrow \pi \nu \nu, \text { peng }}\left(x_{2}, x_{\mu}\right) .
$$

In practice, $f_{\tilde{\chi}^{ \pm}}^{K \rightarrow \pi \nu \nu, \text { peng }}\left(x_{\ell}, x_{2}\right)$ is numerically only $\mathcal{O}(10 \%)$ of $f_{\tilde{\chi}^{ \pm}}^{K \rightarrow \pi \nu \nu, \text { box }}\left(x_{\ell}, x_{2}\right)$, so, out of simplicity, we only present the box contribution for interpreting our results,

$$
\begin{aligned}
f_{\tilde{\chi}^{ \pm}}^{K \rightarrow \pi \nu \nu, \text { box }}\left(x_{\ell}, x_{2}\right)=6\left[\frac{x_{2}\left(x_{2}^{2}-x_{\ell}\right) \log x_{2}}{\left(x_{2}-1\right)^{2}\left(x_{2}-x_{\ell}\right)^{2}}\right. & -\frac{x_{2}}{\left(x_{2}-1\right)\left(x_{2}-x_{\ell}\right)} \\
& \left.-\frac{x_{2} x_{\ell} \log x_{\ell}}{\left(x_{2}-x_{\ell}\right)^{2}\left(x_{\ell}-1\right)}\right]
\end{aligned}
$$

Here $x_{l}=m_{\tilde{l}}^{2} / m_{S}^{2}, x_{2}=M_{2}^{2} / m_{S}^{2}$ and $x_{\mu}=\mu^{2} / m_{S}^{2}$. We have also dropped the neutralino boxes and $Z$-penguins that contribute at the $\mathcal{O}(10 \%)$ level or below.

For $b \rightarrow q \gamma$

$$
\begin{array}{r}
f_{\tilde{\chi}^{ \pm}}^{b \rightarrow s / d \gamma, \text { peng }}\left(x_{\mu}, x_{2}\right)=\frac{6 \sqrt{x_{\mu} x_{2}}}{11}\left[\frac{5 x_{2} x_{\mu}-7 x_{\mu}-7 x_{2}+9}{\left(x_{2}-1\right)^{2}\left(x_{\mu}-1\right)^{2}}+\frac{2\left(2 x_{2}-3\right) \log x_{2}}{\left(x_{2}-1\right)^{3}\left(x_{2}-x_{\mu}\right)}\right. \\
\left.-\frac{2\left(2 x_{\mu}-3\right) \log x_{\mu}}{\left(x_{2}-x_{\mu}\right)\left(x_{\mu}-1\right)^{3}}\right]
\end{array}
$$

Here $x_{\mu}=\mu^{2} / m_{S}^{2}, x_{2}=M_{2}^{2} / m_{S}^{2}$ and $m_{S}$ is the lightest up squark mass eigenvalue. 
For $B_{q} \rightarrow \mu^{-} \mu^{+}$

$$
\begin{aligned}
f_{\tilde{g}}^{B_{q} \rightarrow \mu^{+} \mu^{-}, \mathrm{h} \text {-peng }}\left(x_{q}, x_{g}\right)=\sqrt{x_{g} x_{q}} & {\left[\frac{\left(x_{q}-1\right) x_{g} \log x_{g}}{\left(x_{g}-1\right)^{2}\left(x_{g}-x_{q}\right)}+\frac{1}{\left(x_{g}-1\right)}-\frac{x_{q} \log x_{q}}{\left(x_{q}-1\right)\left(x_{g}-x_{q}\right)}\right] } \\
f_{\tilde{\chi}}^{B_{q} \rightarrow \mu^{+} \mu^{-}, \text {h-peng }}\left(x_{q}, x_{\mu}, x_{2}\right)=\sqrt{x_{2}} & {\left[\frac{x_{2}\left(x_{q}-1\right) \log x_{2}}{\left(x_{2}-1\right)\left(x_{2}-x_{\mu}\right)\left(x_{2}-x_{q}\right)}\right.} \\
& \left.+\frac{x_{\mu}\left(x_{q}-1\right) \log x_{\mu}}{\left(x_{\mu}-x_{2}\right)\left(x_{\mu}-1\right)\left(x_{\mu}-x_{q}\right)}+\frac{x_{q} \log x_{q}}{\left(x_{\mu}-x_{q}\right)\left(x_{2}-x_{q}\right)}\right]
\end{aligned}
$$

Here $x_{g}=m_{\tilde{g}}^{2} / m_{0}^{2}, x_{\mu}=\mu^{2} / m_{0}^{2}, x_{2}=M_{2}^{2} / m_{0}^{2}$ and and $x_{q}=m_{S}^{2} / m_{0}^{2} ; m_{S}$ and $m_{0}$ in this context are the lightest and heaviest squark mass eigenvalues.

Open Access. This article is distributed under the terms of the Creative Commons Attribution License (CC-BY 4.0), which permits any use, distribution and reproduction in any medium, provided the original author(s) and source are credited.

\section{References}

[1] S.P. Martin, A supersymmetry primer, Adv. Ser. Direct. High Energy Phys. 21 (2010) 1 [hep-ph/9709356] [INSPIRE].

[2] G. Isidori, Y. Nir and G. Perez, Flavor physics constraints for physics beyond the standard model, Ann. Rev. Nucl. Part. Sci. 60 (2010) 355 [arXiv: 1002.0900] [InSPIRE].

[3] G.F. Giudice and R. Rattazzi, Theories with gauge mediated supersymmetry breaking, Phys. Rept. 322 (1999) 419 [hep-ph/9801271] [INSPIRE].

[4] ATLAS collaboration, Observation of a new particle in the search for the standard model Higgs boson with the ATLAS detector at the LHC, Phys. Lett. B 716 (2012) 1 [arXiv: 1207.7214] [INSPIRE].

[5] CMS collaboration, Observation of a new boson at a mass of $125 \mathrm{GeV}$ with the CMS experiment at the LHC, Phys. Lett. B 716 (2012) 30 [arXiv:1207.7235] [INSPIRE].

[6] L.J. Hall, D. Pinner and J.T. Ruderman, A natural SUSY Higgs near 126 GeV, JHEP 04 (2012) 131 [arXiv:1112.2703] [InSPIRE].

[7] S. Heinemeyer, O. Stal and G. Weiglein, Interpreting the LHC Higgs search results in the MSSM, Phys. Lett. B 710 (2012) 201 [arXiv:1112.3026] [INSPIRE].

[8] A. Arbey, M. Battaglia, A. Djouadi, F. Mahmoudi and J. Quevillon, Implications of a $125 \mathrm{GeV}$ Higgs for supersymmetric models, Phys. Lett. B 708 (2012) 162 [arXiv:1112.3028] [INSPIRE].

[9] P. Draper, P. Meade, M. Reece and D. Shih, Implications of a $125 \mathrm{GeV}$ Higgs for the MSSM and low-scale SUSY breaking, Phys. Rev. D 85 (2012) 095007 [arXiv:1112.3068] [InSPIRE].

[10] M. Carena, S. Gori, N.R. Shah and C.E.M. Wagner, A $125 \mathrm{GeV}$ SM-like Higgs in the MSSM and the $\gamma \gamma$ rate, JHEP 03 (2012) 014 [arXiv: 1112.3336] [INSPIRE].

[11] J.L. Evans, M. Ibe, S. Shirai and T.T. Yanagida, A $125 \mathrm{GeV}$ Higgs boson and muon $g-2$ in more generic gauge mediation, Phys. Rev. D 85 (2012) 095004 [arXiv:1201.2611] [INSPIRE]. 
[12] Z. Kang, T. Li, T. Liu, C. Tong and J.M. Yang, A heavy SM-like Higgs and a light stop from Yukawa-deflected gauge mediation, Phys. Rev. D 86 (2012) 095020 [arXiv:1203.2336] [INSPIRE].

[13] N. Craig, S. Knapen, D. Shih and Y. Zhao, A complete model of low-scale gauge mediation, JHEP 03 (2013) 154 [arXiv: 1206.4086] [INSPIRE].

[14] A. Albaid and K.S. Babu, Higgs boson of mass $125 \mathrm{GeV}$ in GMSB models with messenger-matter mixing, Phys. Rev. D 88 (2013) 055007 [arXiv:1207.1014] [INSPIRE].

[15] M. Abdullah, I. Galon, Y. Shadmi and Y. Shirman, Flavored gauge mediation, a heavy Higgs and supersymmetric alignment, JHEP 06 (2013) 057 [arXiv: 1209.4904] [INSPIRE].

[16] M.J. Pérez, P. Ramond and J. Zhang, Mixing supersymmetry and family symmetry breakings, Phys. Rev. D 87 (2013) 035021 [arXiv: 1209.6071] [INSPIRE].

[17] H.D. Kim, D.Y. Mo and M.-S. Seo, Neutrino assisted gauge mediation, Eur. Phys. J. C 73 (2013) 2449 [arXiv:1211.6479] [INSPIRE].

[18] P. Byakti and T.S. Ray, Burgeoning the Higgs mass to 125 GeV through messenger-matter interactions in GMSB models, JHEP 05 (2013) 055 [arXiv:1301.7605] [INSPIRE].

[19] N. Craig, S. Knapen and D. Shih, General messenger Higgs mediation, JHEP 08 (2013) 118 [arXiv: 1302.2642] [INSPIRE].

[20] J.A. Evans and D. Shih, Surveying extended GMSB models with $m_{h}=125 \mathrm{GeV}$, JHEP 08 (2013) 093 [arXiv: 1303. 0228] [INSPIRE].

[21] L. Calibbi, P. Paradisi and R. Ziegler, Gauge mediation beyond minimal flavor violation, JHEP 06 (2013) 052 [arXiv:1304.1453] [INSPIRE].

[22] W. Fischler and W. Tangarife, Vector-like fields, messenger mixing and the Higgs mass in gauge mediation, JHEP 05 (2014) 151 [arXiv:1310.6369] [INSPIRE].

[23] V.S. Mummidi and S.K. Vempati, A little more gauge mediation and the light Higgs mass, Nucl. Phys. B 881 (2014) 181 [arXiv:1311.4280] [INSPIRE].

[24] S. Knapen and D. Shih, Higgs mediation with strong hidden sector dynamics, JHEP 08 (2014) 136 [arXiv:1311.7107] [InSPIRE].

[25] R. Ding, T. Li, F. Staub and B. Zhu, Focus point supersymmetry in extended gauge mediation, JHEP 03 (2014) 130 [arXiv:1312.5407] [INSPIRE].

[26] C. Liu and Z.-h. Zhao, A realization of effective SUSY with strong unification, Phys. Rev. D 89 (2014) 057701 [arXiv: 1312.7389] [InSPIRE].

[27] L. Calibbi, A. Mariotti, C. Petersson and D. Redigolo, Selectron NLSP in gauge mediation, JHEP 09 (2014) 133 [arXiv: 1405.4859] [INSPIRE].

[28] A. Basirnia, D. Egana-Ugrinovic, S. Knapen and D. Shih, 125 GeV Higgs from tree-level A-terms, JHEP 06 (2015) 144 [arXiv: 1501.00997] [InSPIRE].

[29] Z. Chacko and E. Ponton, Yukawa deflected gauge mediation, Phys. Rev. D 66 (2002) 095004 [hep-ph/0112190] [INSPIRE].

[30] Y. Shadmi and P.Z. Szabo, Flavored gauge-mediation, JHEP 06 (2012) 124 [arXiv: 1103.0292] [INSPIRE].

[31] J.L. Evans, M. Ibe and T.T. Yanagida, Relatively heavy Higgs boson in more generic gauge mediation, Phys. Lett. B 705 (2011) 342 [arXiv:1107.3006] [INSPIRE]. 
[32] I. Galon, G. Perez and Y. Shadmi, Non-degenerate squarks from flavored gauge mediation, JHEP 09 (2013) 117 [arXiv:1306.6631] [INSPIRE].

[33] T. Jelinski and J. Pawelczyk, Masses and FCNC in flavoured GMSB scheme, arXiv:1406.4001 [INSPIRE].

[34] L. Calibbi, P. Paradisi and R. Ziegler, Lepton flavor violation in flavored gauge mediation, Eur. Phys. J. C 74 (2014) 3211 [arXiv:1408.0754] [InSPIRE].

[35] J.A. Evans and D. Shih. The SUSY CP problem in EGMSB models, to appear.

[36] T. Hahn, Generating Feynman diagrams and amplitudes with FeynArts 3, Comput. Phys. Commun. 140 (2001) 418 [hep-ph/0012260] [INSPIRE].

[37] T. Hahn and M. Pérez-Victoria, Automatized one loop calculations in four-dimensions and D-dimensions, Comput. Phys. Commun. 118 (1999) 153 [hep-ph/9807565] [INSPIRE].

[38] W. Porod, F. Staub and A. Vicente, A flavor kit for BSM models, Eur. Phys. J. C 74 (2014) 2992 [arXiv: 1405.1434] [INSPIRE].

[39] J.A. Evans and D. Shih. FormFlavor v1.0: manual and validation, to appear.

[40] A. Lenz, B-mixing in and beyond the Standard model, arXiv:1409.6963 [INSPIRE].

[41] J. Brod, M. Gorbahn and E. Stamou, Two-loop electroweak corrections for the $K \rightarrow \pi \nu \overline{n u}$ decays, Phys. Rev. D 83 (2011) 034030 [arXiv: 1009.0947] [inSPIRE].

[42] M. Misiak and M. Steinhauser, NNLO QCD corrections to the $\bar{B} \rightarrow X_{s} \gamma$ matrix elements using interpolation in $m_{c}$, Nucl. Phys. B 764 (2007) 62 [hep-ph/0609241] [INSPIRE].

[43] BaBAr collaboration, P. del Amo Sanchez et al., Study of $B \rightarrow X \gamma$ decays and determination of $\left|V_{t d} / V_{t s}\right|$, Phys. Rev. D 82 (2010) 051101 [arXiv:1005.4087] [INSPIRE].

[44] A. Crivellin and L. Mercolli, $B \rightarrow X_{d} \gamma$ and constraints on new physics, Phys. Rev. D 84 (2011) 114005 [arXiv: 1106.5499] [INSPIRE].

[45] CMS AND LHCb collaboration, Combination of results on the rare decays $B_{(s)}^{0} \rightarrow \mu^{+} \mu^{-}$ from the CMS and LHCb experiments, LHCb-CONF-2013-012 (2013) [CERN-LHCb-CONF-2013-012].

[46] C. Bobeth et al., $B_{s, d} \rightarrow \ell^{+} \ell^{-}$in the standard model with reduced theoretical uncertainty, Phys. Rev. Lett. 112 (2014) 101801 [arXiv:1311.0903] [InSPIRE].

[47] Particle Data Group collaboration, J. Beringer et al., Review of particle physics, Phys. Rev. D 86 (2012) 010001 [INSPIRE].

[48] Heavy Flavor Averaging Group collaboration, Y. Amhis et al., Averages of B-hadron, C-hadron and $\tau$-lepton properties as of early 2012, arXiv:1207.1158 [INSPIRE].

[49] W. Altmannshofer, A.J. Buras, S. Gori, P. Paradisi and D.M. Straub, Anatomy and phenomenology of FCNC and CPV effects in SUSY theories, Nucl. Phys. B 830 (2010) 17 [arXiv: 0909.1333] [INSPIRE].

[50] W. Altmannshofer, R. Harnik and J. Zupan, Low energy probes of PeV scale sfermions, JHEP 11 (2013) 202 [arXiv: 1308.3653] [INSPIRE].

[51] J.A. Evans and D. Shih. Chiral flavor violation, to appear.

[52] A.J. Buras, S. Jager and J. Urban, Master formulae for $\Delta F=2$ NLO QCD factors in the standard model and beyond, Nucl. Phys. B 605 (2001) 600 [hep-ph/0102316] [INSPIRE]. 
[53] B. Grinstein, R.P. Springer and M.B. Wise, Strong interaction effects in weak radiative $\bar{b}$ meson decay, Nucl. Phys. B 339 (1990) 269 [INSPIRE].

[54] M. Misiak et al., Updated NNLO QCD predictions for the weak radiative B-meson decays, Phys. Rev. Lett. 114 (2015) 221801 [arXiv:1503.01789] [INSPIRE].

[55] C. Bobeth, T. Ewerth, F. Krüger and J. Urban, Enhancement of $B\left(\bar{B}_{d} \rightarrow \mu^{+} \mu^{-}\right) / B\left(\bar{B}_{s} \rightarrow \mu^{+} \mu^{-}\right)$in the MSSM with minimal flavor violation and large $\tan \beta$, Phys. Rev. D 66 (2002) 074021 [hep-ph/0204225] [inSPIRE].

[56] A. Dedes, J. Rosiek and P. Tanedo, Complete one-loop MSSM predictions for $B \rightarrow \ell^{+} \ell^{-}$at the Tevatron and LHC, Phys. Rev. D 79 (2009) 055006 [arXiv:0812.4320] [InSPIRE].

[57] T. Hermann, M. Misiak and M. Steinhauser, Three-loop QCD corrections to $B_{s} \rightarrow \mu^{+} \mu^{-}$, JHEP 12 (2013) 097 [arXiv: 1311.1347] [INSPIRE].

[58] J. Brod and M. Gorbahn, Next-to-next-to-leading-order charm-quark contribution to the CP-violation parameter $\epsilon_{K}$ and $\Delta M_{K}$, Phys. Rev. Lett. 108 (2012) 121801 [arXiv: 1108.2036] [INSPIRE].

[59] RBC, UKQCD collaboration, N.H. Christ, T. Izubuchi, C.T. Sachrajda, A. Soni and J. Yu, Long distance contribution to the KL-KS mass difference, Phys. Rev. D 88 (2013) 014508 [arXiv: 1212.5931] [INSPIRE].

[60] Z. Bai et al., $K_{L}-K_{S}$ mass difference from lattice QCD, Phys. Rev. Lett. 113 (2014) 112003 [arXiv: 1406.0916] [INSPIRE].

[61] Quark Flavor Physics Working Group collaboration, J.N. Butler et al., Working group report: quark flavor physics, arXiv:1311.1076 [INSPIRE].

[62] Belle-II collaboration, T. Abe et al., Belle II technical design report, arXiv:1011.0352 [INSPIRE].

[63] T. Aushev et al., Physics at super B factory, arXiv:1002.5012 [INSPIRE].

[64] NA62 collaboration, NA62 technical design report, NA62-10-07 (2010).

[65] NA62 collaboration, R. Fantechi, The NA62 experiment at CERN: status and perspectives, arXiv: 1407.8213 [INSPIRE].

[66] ORKA collaboration, E.T. Worcester, ORKA, the golden kaon experiment: precision measurement of $K^{+} \rightarrow \pi^{+} \nu \bar{\nu}$ and other rare processes, PoS (KAON13) 035 [arXiv:1305.7245] [INSPIRE].

[67] RBC, UKQCD collaboration, N. Christ, T. Izubuchi, C.T. Sachrajda, A. Soni and J. Yu, Calculating the $K_{L}-K_{S}$ mass difference and $\epsilon_{K}$ to sub-percent accuracy, PoS(LATTICE 2013) 397 [arXiv: 1402 .2577] [INSPIRE].

[68] R. Mahbubani, M. Papucci, G. Perez, J.T. Ruderman and A. Weiler, Light nondegenerate squarks at the LHC, Phys. Rev. Lett. 110 (2013) 151804 [arXiv:1212.3328] [INSPIRE].

[69] M. Blanke, G.F. Giudice, P. Paradisi, G. Perez and J. Zupan, Flavoured naturalness, JHEP 06 (2013) 022 [arXiv: 1302.7232] [INSPIRE].

[70] P. Agrawal and C. Frugiuele, Mixing stops at the LHC, JHEP 01 (2014) 115 [arXiv: 1304.3068] [INSPIRE].

[71] M. Backović, A. Mariotti and M. Spannowsky, Signs of tops from highly mixed stops, JHEP 06 (2015) 122 [arXiv:1504.00927] [INSPIRE]. 
[72] J.-h. Park, Metastability bounds on flavour-violating trilinear soft terms in the MSSM, Phys. Rev. D 83 (2011) 055015 [arXiv: 1011.4939] [INSPIRE].

[73] M. Arana-Catania, S. Heinemeyer and M.J. Herrero, Updated constraints on general squark flavor mixing, Phys. Rev. D 90 (2014) 075003 [arXiv:1405.6960] [inSPIRE].

[74] K. Kowalska, Phenomenology of SUSY with general flavour violation, JHEP 09 (2014) 139 [arXiv: 1406.0710] [INSPIRE].

[75] D.M. Pierce, J.A. Bagger, K.T. Matchev and R.-J. Zhang, Precision corrections in the minimal supersymmetric standard model, Nucl. Phys. B 491 (1997) 3 [hep-ph/9606211] [INSPIRE].

[76] B.C. Allanach et al., SUSY Les Houches accord 2, Comput. Phys. Commun. 180 (2009) 8 [arXiv:0801.0045] [INSPIRE].

[77] S. Aoki et al., Review of lattice results concerning low-energy particle physics, Eur. Phys. J. C 74 (2014) 2890 [arXiv:1310.8555] [INSPIRE].

[78] T. Bae et al., Beyond the standard model b-parameters with improved staggered fermions in $N_{f}=2+1 Q C D$, arXiv:1310.7372 [INSPIRE].

[79] ETM collaboration, N. Carrasco et al., $K$ and $D$ oscillations in the Standard Model and its extensions from $N_{f}=2+1+1$ Twisted Mass LQCD, PoS(LATTICE 2013) 393 [arXiv: 1310.5461] [INSPIRE].

[80] ETM collaboration, N. Carrasco et al., B-physics computations from $N_{f}=2 \operatorname{tm} Q C D$, PoS (LATTICE 2013) 382 [arXiv: 1310.1851] [INSPIRE].

[81] A.J. Buras, Weak Hamiltonian, CP-violation and rare decays, hep-ph/9806471 [INSPIRE].

[82] V. Cirigliano, G. Ecker, H. Neufeld, A. Pich and J. Portoles, Kaon decays in the standard model, Rev. Mod. Phys. 84 (2012) 399 [arXiv:1107.6001] [InSPIRE].

[83] A.J. Buras, A. Czarnecki, M. Misiak and J. Urban, Completing the NLO QCD calculation of $\bar{B} \rightarrow X_{s} \gamma$, Nucl. Phys. B 631 (2002) 219 [hep-ph/0203135] [INSPIRE].

[84] T. Hurth, E. Lunghi and W. Porod, Untagged $\bar{B} \rightarrow X_{s+d} \gamma C P$ asymmetry as a probe for new physics, Nucl. Phys. B 704 (2005) 56 [hep-ph/0312260] [INSPIRE].

[85] F. Staub, Automatic calculation of supersymmetric renormalization group equations and self energies, Comput. Phys. Commun. 182 (2011) 808 [arXiv: 1002.0840] [INSPIRE].

[86] W.J. Marciano and Z. Parsa, Rare kaon decays with 'missing energy', Phys. Rev. D 53 (1996) 1 [INSPIRE].

[87] C.W. Bauer, Z. Ligeti, M. Luke, A.V. Manohar and M. Trott, Global analysis of inclusive B decays, Phys. Rev. D 70 (2004) 094017 [hep-ph/0408002] [InSPIRE]. 